\title{
Genetic dissection of photosynthetic performances in maize under drought-stressed and well-watered environments
}

\section{Xiaoqiang Zhao ( $\sim$ zhaoxq3324@163.com )}

Gansu Provincial Key Laboratory of Aridland Crop Science, Gansu Agricultural University https://orcid.org/0000-0003-1255-5006

\section{Yantian Lu}

Gansu Provincial Key Lab of Aridland Crop Science, Gansu Agricultural University

\section{Mingxing Bai}

Gansu Provincial Key Lab of Aridland Crop Science, Gansu Agricultural University

\section{Wenli Li}

Gansu Provincial Key Lab of Aridland Crop Science, Gansu Agricultural University

\section{Dan Zhang}

Gansu Provincial Key Lab of Aridland Crop Science, Gansu Agricultural University

\section{Guichen Li}

Gansu Provincial Key Lab of Aridland Crop Science, Gansu Agricultural University

\section{Yuan Zhong}

Gansu Provincial Key Lab of Aridland Crop Science, Gansu Agricultural University

\section{Research article}

Keywords: Maize (Zea mays L.), QTLs, Photosynthetic performances, Drought, Candidate genes

Posted Date: December 16th, 2019

DOI: https://doi.org/10.21203/rs.2.18926/v1

License: (c) (1) This work is licensed under a Creative Commons Attribution 4.0 International License. Read Full License 
1 Genetic dissection of photosynthetic performances in maize under

2 drought-stressed and well-watered environments

4 Xiaoqiang Zhao*, Yantian Lu, Mingxing Bai, Wenli Li, Dan Zhang, Guichen Li, Yuan

5 Zhong*

\section{Abstract}

Background: Maintaining photosynthetic capacities is a critical function that allows maize (Zea mays

8 L.) to adapt to drought stress. The elucidation of genetic controls of photosynthetic performances, and tightly linked molecular markers under water stress are thus of great importance in marker-assisted selection (MAS) breeding. Meanwhile, little is known regarding their genetic controls under drought stress. Two $\mathrm{F}_{4}$ populations were developed to identify quantitative trait loci (QTLs) and dissect the genetic variation underlying six photosynthetic-related traits, namely, net photosynthetic rate (Pn), stomatal conductance $(\mathrm{Gs})$, intercellular $\mathrm{CO}_{2}$ concentration $(\mathrm{Ci})$, transpiration rate $(\mathrm{Tr})$, ribulose 1,5 biphosphate carboxylase activity (RuBP), and water use efficiency (WUE) under drought-stressed and well-watered environments.

Results: For two populations, we detected 54 QTLs under drought-stressed and well-watered environments by single-environment mapping with composite interval mapping (CIM), approximately 81.8 100 \% QTLs displayed non-additive effects, and 43 of the 54 QTLs were identified under droughtstressed environment. We also dissected 54 QTLs via joint analysis of all environments with mixedlinear-model-based composite interval mapping (MCIM), 24 QTLs involved in QTL $\times$ environment interactions (QEIs), approximately $87.5 \%$ QEIs were identified under drought-stressed environments, as well as 14 pair epistasis exhibited dominance-by-additive/dominance (DA/DD) effects under constracting environments. We further identified 8 constitutive QTLs (cQTLs) across two populations by CIM/MCIM under multiple environments. Remarkably, bin 1.07_1.10 (cQTL2), bin 6.05 (cQTL5), bin 7.02_7.04 (cQTL6), bin 8.03 (cQTL7), and bin 10.03 (cQTL8) exhibited 5 pleiotropic cQTLs that were consistent with phenotypic correlations among all photosynthetic-related traits. Additionally, 17 candidate genes were validated in above cQTLs.

Conclusions: Photosynthetic performances in maize were predominantly controlled by non-additive and QEIs effects, where more QEIs effects occurred in drought stress. 8 cQTLs affecting six photosyntheticrelated traits could be useful for genetic improvement of these traits via QTL pyramiding, corresponding 5 QTLs clusters indicated tight linkage or pleiotropy in the inheritance of these traits, and 17 candidate genes involved in leaf morphology and development, photosynthesis, and stress reponse coincided with above corresponding cQTLs.

34 Keywords: Maize (Zea mays L.); QTLs; Photosynthetic performances; Drought; Candidate genes

\section{Background}

\footnotetext{
* Correspondence: zhaoxq3324@163.com; zhongy@gsau.edu.cn

Gansu Provincial Key Lab of Aridland Crop Science, Gansu Agricultural University, Lanzhou 730070, China
} 
Water stress is one of the most important environmental limiting factors for maize (Zea mays L.) productivity in tropical and subtropical regimes [1], and the global climate change scenario and growing population exerts great pressure tend to increase the problems of food insecurity. Therefore, the improvement of resistance to water scarcity and breeding drought tolerant varieties are crucial for maize surivival, growth, and biomass production living in water scarce environments [2-3]. Maize as an important $\mathrm{C}_{4}$ crop, and its highly complex mechanism of phoptosynthetic performance is one of the main targets for improving maize grain yield (GY) and drought resistance. He et al. [4], Liu et al. [5] and Zhao et al. [6] reported that net ohotosynthetic rate (Pn), chlorophyll relative content (SPAD), chlorophyll a content (FCa), chloroohyll b content ( $\mathrm{FCb}$ ), total chlorophyll content $(\mathrm{FCt})$, ribulose 1,5-biphosphate carboxylase activity (RuBP), stomatal conductance (Gs), and transpiration rate ( $\mathrm{Tr}$ ) in maize were significantly reduced, meanwhile chloroohy a/b $(\mathrm{FCa} / \mathrm{b})$, intercellular $\mathrm{CO}_{2}$ concentration $(\mathrm{Ci})$ and water use effeciency (WUE) were significantly increased under drought stress, and compared with drought sensitive materials, stronge drought resistant maize could maintain higher photosynthetic capacity in drought land. Zhang et al. [7] suggested moderate and severe drought stress caused an obvious decrease in Pn, Gs, and RuBP, caused a significant increase in Ci, damaged photosytem II (PSII), reduced electron transport in diverse maize varieties. Tezara et al. [8] and Liu et al. [9] also analyzed the activities of photosystem I (PSI), PSII, and photosynthetic electron transport chain (PETC) between PSII and PSI in response to drought stress in maize, and of which showed that a significant increase in absorption of antenna chlorophyll per PSII reaction center (ABS/RC) was found, a significant decrease in quantum yield for electron transport $\left(\varphi E_{0}\right)$, efficiency of an electron beyond primary quinone acceptor of PSII $\left(\mathrm{Q}_{\mathrm{A}}\right)$, efficiency of an electron beyond $\mathrm{Q}_{\mathrm{A}}\left(\Psi_{0}\right)$, and performance index for energy conservation from photons absorbed by PSII to the reduction of intersystem electron acceptors $\left(\mathrm{PI}_{\mathrm{ABS}}\right)$ were observed, and no significant change in the fast $820 \mathrm{~nm}$ modulated reflection (MR) phase and the amplitude of delayed fluorescence under drought stress. In this regard, once photosynthesis performance is inhibited under drought environments, it is likely to result in maize plant absorbing more light energy than can be consumed through photosynthetic carbon fixation, and even damage photo-oxidation, finally cause more GY losses.

Using identified molecular markers and quantitative trait loci (QTLs) for photosynthetic-related traits under different watering treatments in marker-assisted selection (MAS) breeding is a promising way to maximize the productivity of maize grown in droght land. Until now, genetic studies on wheat (Triticum aestivum L.) [10], rice (Oryza sativa L.) [11-13], sunflower (Helianthus annuus L.) [14], rapeseed (Brassica napus L.) [15], cotton (Gossypium spp) [16-17] etc. for photosynthetic-related traits have been extensively conducted using QTL mapping during drought, high temperature, salt and alkali stresses. In maize, however, only several experiments have been conducted for mapping QTLs for photosyntheticrelated traits, e.g., Wang and Zhang [18] mapped 32 QTLs for FCa, FCb, FCt, and other chlorophyll content $(\mathrm{FCc})$ in one $\mathrm{F}_{2}$ population derived from A150-3-2 $\times$ Mo17. Trachsel et al. [19] found 7 QTLs revalated to quantum efficiency of photosystem II (ФPSII), and SPAD in the intermated CML444 $\times$ SCMalawi recombinant inbred lines (RILs) populations. Yu et al. [20] detected 32 QTLs associated with $\mathrm{FCa}, \mathrm{FCb}, \mathrm{FCt}, \mathrm{Pn}, \mathrm{Gs}, \mathrm{Ci}$, and $\mathrm{Tr}$ in two $\mathrm{F}_{2}$ populations derived from Y114 $\times$ Y115 and Y105 $\times$ Y 106 . In addition, only two mapping studies for photosynthetic performance in maize have been reported under 
drought environments, e.g., Pelleschi et al. [21] identified 19 major QTLs controlling net $\mathrm{CO}_{2}$-uptake (CO), ADPglucose pyrophosphorylase (AGP), Gs, and Tr in a RILs population derived from the cross F$2 \times$ MBS847 under drought-stressed and well-watered regimes. Prado et al. [22] also assessed 16 robust QTLs affecting Gs with a diversity panel of maize hybrids under water dificit. Even the photosyntheticrelated ZmPPDK, i.e., pyruvate, orthophosphate dikinase 1 gene, the transgenic wheat [23], potato (Solanum tuberosum) [24], and Arabidopsis thaliana [25] plants with ZmPPDK could significantly improve their photosynthetic efficiency and GY. Despite these researches, the molecular mechanisms for photosynthetic-related trait in response to drought remains poorly understood. Thus, unraveling in-depth investigations on the genetic mechanisms controlling for photosynthetic performances in contrasting watering treatments, which may be used to speculate the possible genetic locations of corresponding candidate genes. Moreover, a better understanding of the genotype $\times$ environment interaction (GEI) will provide a foundation for the genetic improvement and optimization of genotypes across different environments [26].

The overall objective of this study were to identify QTLs responsible for Pn, Gs, Ci, Tr, RuBP, and WUE in two maize $\mathrm{F}_{4}$ populations subjected to both watering regimes as well as estimate their effects by single environment mapping with composite interval mapping (CIM), then to further dissect joint QTLs, QTL by QTL interaction (epistasis), as well as QTL $\times$ environment interactions (QEIs) among all watering environments via mixed-linear-model based composite interval mapping (MCIM). By combining these experiments, we hope to identify hot spots, areas of co-localization experiments, as well as corresponding candidate genes, which may further contribute to mapping QTLs, revealing genetic mechanisms for photosynthetic performances under diverse watering environments, and developing maize MAS breeding for photosynthetic performances to improve drought tolerance and GY in the future.

\section{Methods}

\section{Plant materials}

The two $\mathrm{F}_{4}$ mapping populations included 218 and 202 families (POP-CT and POP-LT) derived from Chang7-2 $\times$ TS141 $(\mathrm{CH} \times \mathrm{TS})$ and Langhuang $\times$ TS141 $(\mathrm{LH} \times \mathrm{TS})$ hybrids. The corresponding two $\mathrm{F}_{2}$ families derived from the corresponding crosses self-pollinated to generate $F_{3}$ families at Pingliang maize breeding station of Gansu Agricultural University, China $\left(106.93^{\circ} \mathrm{N}, 35.43^{\circ} \mathrm{E} ; 1,204 \mathrm{~m}\right.$ altitude; loessial soil) in 2013, and then each $F_{3}$ plant was again selfed to derive the corresponding $F_{4}$ families at Zhangye breeding base of Yuyuan Co., LTD., China $\left(38.83^{\circ} \mathrm{N}, 106.93^{\circ} \mathrm{E} ; 1,785 \mathrm{~m}\right.$, altitude; sandloam) in 2015 and Jingtai farm of Tiaoshan Nongken corporation, China $\left(37.18^{\circ} \mathrm{N}, 104.03^{\circ} \mathrm{E} ; 1,640 \mathrm{~m}\right.$, altitude; sandloam) in 2015, respectively [27]. In addition, Chang7-2 (drought-tolerant line), Langhuang (droughttolerant line), and TS141 (drought-sensitive line) are foundation inbred lines in China maize breeding and are representatives of Tangsipingtou (TSPT), TSPT, and Reid yellow dent (Reid) heterotic groups, respetively [28-29].

\section{Field experiment and trait evaluation}

The phenotype data of the two $\mathrm{F}_{4}$ families (POP-CT and POP-LT) and their parents (CH, LT, and TS) were evaluated in a completely randomized block design with two replications and 10 plants at a density 
of 55,580 plant ha $^{-1}$ for each plot at Huangyang, Gansu Academy of Agricultural Sciences Proving Groud, China (http://hyc.gsagr.ac.cn/channels/channel_503_1.html) $\left(37.67^{\circ} \mathrm{N}, 102.85^{\circ} \mathrm{E} ; 1,740 \mathrm{~m}\right.$, altitude; sandloam) in 2019. The mean temperature, total sunshine duration, total rainfall, total evaporation capacity, mean relative humidity, and mean wind velocity at the experimental site was $17.9{ }^{\circ} \mathrm{C}, 1,427.6$ $\mathrm{h}, 118.7 \mathrm{~mm}, 1,039.7 \mathrm{~mm}, 52.3 \%$, and $2.2 \mathrm{~m} \mathrm{~s}^{-1}$ during the growing seasons (from April to September) in 2019, respectively (Additinal file 1: Fig. S1). Then the experimental field was divided into wellwatered (WW) and drought-stressed (DS) treatments. The DS treatment was equivalent to rainfed conditions with rainfall of $118.7 \mathrm{~mm}$ during the growing season (from April to September). The WW treatment involved irrigation with $4,500 \mathrm{~m}^{3} \mathrm{ha}^{-1}$ water supply at each of V18, R1, and R3 stage. In addition, because of the abundant annual evaporation capacity $(2,164.7 \mathrm{~mm})$, low annual relative humidity $(45.8 \%)$, scarce annual rainfall $(172 \mathrm{~mm})$, and low annual mean temperature $\left(9.3{ }^{\circ} \mathrm{C}\right)$ in this experimental site (Additinal file 1: Fig. S1), plastic film ( $0.02 \mathrm{~mm}$ thick, $140 \mathrm{~cm}$ wide) was laid out by land over the fileds and covered the soil surface before sowing.

Until spreading pollen stage, the six photosynthetic-related traits, i.e., $\mathrm{Pn}\left(\mu \mathrm{mol} \mathrm{CO}_{2} \cdot \mathrm{m}^{-2} \cdot \mathrm{s}^{-1}\right)$, Gs (mol $\left.\mathrm{H}_{2} \mathrm{O} \cdot \mathrm{m}^{-2} \mathrm{~s}^{-1}\right), \mathrm{Ci}\left(\mu \mathrm{mol} \mathrm{CO} \mathrm{Col}_{2} \cdot \mathrm{mol}^{-1}\right), \operatorname{Tr}\left(\mathrm{mol} \mathrm{H}_{2} \mathrm{O} \cdot \mathrm{m}^{-2} \mathrm{~s}^{-1}\right), \operatorname{RuBP}\left(\mathrm{mol} \cdot \mathrm{m}^{-2 \cdot} \mathrm{s}^{-1}\right)$, and WUE $\left(\mu \mathrm{mol} \mathrm{CO}_{2} / \mathrm{mol}\right.$ $\mathrm{H}_{2} \mathrm{O}$ ) were measured in the corresponding populations and parents, using a portable photosynthesis system, namely LI-6400 XT (LI-COR Inc. Lincoln, Nebraska, USA). Specifically, Pn, Gs, Ci, and Tr were measured at a light intensity of $1,500 \mu \mathrm{mol} \mathrm{m}^{-2} \mathrm{~s}^{-1}$ photosynthetic active radiation (PAR), a leaf temperature of $30{ }^{\circ} \mathrm{C}$ and a constant $\mathrm{CO}_{2}$ concentration of $380 \pm 5 \mu \mathrm{mol} \mathrm{CO}_{2} \mathrm{~mol}^{-1}$ in the sample chamber provided with buffer volume [13]. All measurements for each treatment were made on the fully expanded leaves between 9:00 and 11:00 a.m. on sunny days to avoid effects of photo-inhibition and were repeated at least 5 times using different plants. RuBP was estimated in response to apparent mesophyll conductance (AMC) as follows [3]: $\mathrm{RuBP}=\mathrm{Pn} / \mathrm{Ci}$ (1). And WUE was also calculated as follows [10, 17]: WUE $=\operatorname{Pn} / \operatorname{Tr}(2)$. Then the corresponding $F_{4}$ plants were harvested and subsequently air-dried to evaluated ear weight (EW), grain weight per ear (GW), and 100-kernel weight (KW). Relative to the WW treatment, the average rate of change (RC) of each trait under DS treatment was also estimated according to Zhao et al. [28] as follows: $R C=\left(1-T_{D} / T_{W}\right) \times 100 \%$ (3). Here, $T_{D}$ was the average value of each trait under DS treatment, and $\mathrm{T}_{\mathrm{W}}$ was the average value of corresponding trait under WW treatment.

\section{Statistical analysis of phenotypic data}

144 The all phenotypic data obtained from two $\mathrm{F}_{4}$ populations and three parents under each watering environment were statistically analyzed using IBM-SPSS Statistics v. 19.0 (SPSS Inc., Chicago, IL, USA) (https://www.ibm.com/products/spss-statistics). The significance of total and residual variances of each trait in two $\mathrm{F}_{4}$ populations were estimated by the general linear model for univariate (GLM-Univariate) with one-way analysis of variance (ANOVA), respectively. The broad-sense heritability $\left(h^{2}\right)$ and GEI heritability $\left(h_{g e}^{2}\right)$ for combined environments (i.e., WW and DS) were estimated as follows [30]: $h^{2}=$ $\sigma_{g}^{2} /\left(\sigma_{g}^{2}+\sigma_{g e}^{2} / n+\sigma_{\varepsilon}^{2} / \mathrm{nr}\right)(4)$ and $h_{g e}^{2}=\left(\sigma_{g e}^{2} / n\right) /\left(\sigma_{g}^{2}+\sigma_{g e}^{2} / n+\sigma_{e}^{2} / \mathrm{nr}\right)(5)$. Here, $\sigma_{g}^{2}$ was genotypic variance, $\sigma_{e}^{2}$ was environmental variance, $\sigma_{\varepsilon}^{2}$ was error variance, $\sigma_{g e}^{2}$ was GEI variance, $\mathrm{n}(\mathrm{n}=2)$ was the No. of environments, and $r$ was the No. of replications for the experiment $(r=10)$. Possible 
associations between corresponding traits were tested using several methods, based either on phenotypic Pearson correlation or on principal component Analysis (PCA) via IBM-SPSS Statistics v. 19.0 [21].

\section{Genetic map construction and QTLs identification}

A total of 205 and 199 genome-wide polymorphic simple sequence repeats (SSRs) obtained from the Maize Genetics and Genomics Database (MaizeGDB, http://www.maizegdb.org/) were applied to develop two $F_{2}$ populations genetic maps, using JionMap v. 4.0 (https://www.kyazma.nl/index.php/ JoinMap/), and the total maps length were $1,648.8$ and $1,542.5 \mathrm{cM}$, with an average interval of 8.0 and $7.8 \mathrm{cM}$, respectively [27-29].

In a single watering environment, QTL mapping for each photosynthetic-related trait was performed using CIM implemented with Windows QTL Cartographer software v. 2.5 (http://statgen.ncsu.edu/ qtlcart/winqtlcart.htm). For CIM, model 6 of the Zmapqtl module was used to analyze QTLs. Window size was $10 \mathrm{cM}$, and cofactors were selected through forward and backward regressions with the in and out thresholds at a $P<0.05$. A genome-wide critical threshold value was estimated for experiment-wise type I error rate of 0.05 with 1,000 random permutations [31]. The identified QTLs effects were estimated according to criteria suggested by Stuber et al. [32] as follows: |dominance/additive| (|d/a|); additive (A), $|\mathrm{d} / \mathrm{a}|=0.00 \sim 0.20$; partial dominance (PD), $|\mathrm{d} / \mathrm{a}|=0.21 \sim 0.80$; dominance (D), $|\mathrm{d} / \mathrm{a}|=0.81 \sim 1.20$; overdomiance (OD), $|\mathrm{d} / \mathrm{a}|>1.20$.

Additionally, among all watering environments, the MCIM via QTL Network v. 2.0 (http://ibi.zju.edu.cn/software/qtlnetwork) was used to dissect joint QTLs, epistatic QTLs, and QEI for each photosynthetic-related trait based on the all watering environments. The testing window, walk speed, and filtration window of the genome scan were set at 10,2, and $10 \mathrm{cM}$, respectively. A total of 1,000 permutations were performed to determine the threshold logarithm (base 10) of odds ratio (LOD), for the traits for declaring a significant QTL at a $P<0.05$ probability level. The name of QTL was assigned according to the modifying nomenclature of Zhao et al. [29]. Furthermore, the letter "J" was added into the middle of the QTL name (i.e., inserted between trait abbreviation and No. of chromosome), whereas one QTL was only detected in joint analysis with MCIM but not in a single environment through the CIM program. The LOD confidence interval (CI) of the QTL were estimated according to Zhao et al. [26] as follows: $\mathrm{CI}=530 /\left(\mathrm{N} \times \mathrm{R}^{2}\right)$ (6). Here, $\mathrm{N}$ was the population size, $\mathrm{R}^{2}$ was the value of the phenotypic variation contributed by the QTL. Additionally, the photosynthetic-related traits QTLs were identified within same marker interval or within overlapping Cis, the corresponding loci were assumed to be common QTLs with pleiotropic effects.

\section{Constitutive QTLs (cQTLs) detection and candidate genes dissection}

cQTLs refer to the QTL stably and repeatedly detected with CIM/MCIM across different mapping populations under two or more watering environments [33]. Then the corresponding cQTLs intervals were projected on the physical reference map B73 RefGen_v4 (https://maizegdb.org/gbrowse/maize_v4), and the corresponding candidate genes were further validated in corresponding cQTL regimes [34], and corresponding genes functions of which even were analyzed via the AgBase v. 2.00 (http://agbase.arizona. edu/) online software and public databases, namely, National Center for Biotechnology Information 

National Knowledge Infrastructure (CNKI, http://www.cnki.net).

\section{3}

194

\section{Results}

\section{Photosynthetic performances variations under drought-stressed environments}

We analyzed the six tested photosynthetic performance values across three parents and two $\mathrm{F}_{4}$ populations under both experimental watering treatments. There were significance difference at $P<0.05$ or $P<0.01$ level on six photosynthetic-related traits in three parents under well-watered and droughtstressed environments (Additional file 2: Table S1). Compared with well-watered environment, the Pn, Gs, Tr, and RuBP showed significant decreases in Chang7-2, Langhuang, TS141, POP-CT, and POP-LT, $\mathrm{Ci}$ and WUE, however, displayed significant increases under drought-stressed environments (Fig. 1). Further analysis indicated that drought-sensitive line TS141 had larger RC in Pn, Gs, Ci, and RuBP, but had less RC in Tr compared to drought-tolerant lines Chang7-2 and Langhuang, and even the average $\mathrm{RC}$ of the three parents and two $\mathrm{F}_{4}$ populations in Pn, Gs, Ci, Tr, RuBP, and WUE were 16.02, 17.05, $9.34,10.80,18.96$, and $-8.62 \%$, respectively (Fig. 1). These phenotypic analyses demonstrated that the drought-induced limitation of photosynthesis in maize was primarily due to $\mathrm{CO}_{2}$ diffusion efficiency from sub-stomatal interval internal cavities to carboxylation site in chloroplasts and degree of stomatal closure (SC), therefore, the RuBP and Gs decreased remarkably in different maize materials under drought stress. Moreover, except for RuBP, other measured photosynthetic performance values in two $\mathrm{F}_{4}$ populations were continuously distributed, with absolute values of skewness and kurtosis being less than 1.0 (Additional file 2: Table S1, Additional file 3: Fig. S2), indicative of continuous variation and a quantitative genetic basis in these maize photosynthetic performances. For all photosynthetic performances in two $\mathrm{F}_{4}$ populations, results of ANOVA showed that there were significant variation among genotypes (Table 1), which further allowed their genetic dissection under drought stress. The environmental and GEI variances were also significant $(P<0.01$ or $P<0.05)$ (Table 1), which implied that the populations performed quite inconsistently between the both watering environments. Additionally, the estimated $h^{2}$ and $h_{g e}^{2}$ values of the all photosynthetic performance in two populations were 46.429 (Gs in POP-CT) 91.030 \% (Ci in POP-LT)/4.531 (Ci in POP-LT) 19.770 \% ( $\mathrm{Tr}$ in POP-CT) because of the large GEI contribution to phenotypic variances (Table 1).

\section{Phenotypic framework of photosynthetic performances}

Examination of phenotypic correlations between photosynthetic-related traits may be usefull before interpreting the co-locations between QTLs which were more likely to reveal genetic relationships. PCA and Pearson correlations were performed in two populations and three parents under both watering environments. PCA, under well-watered and drought-stressed environment, displayed two significant PCs (PC1 and PC2) with eigenvalues greater than 1 were extracted that together explained $65.032 \%$ and $75.602 \%$ of the variance, respectively (Fig. 2). These PCs were linear combinations of the original photosynthetic-related traits that were independent of each other, and represented different combinations of the traits based on their variable loadings under different watering environments. Among them, PC1 primarily represented variances in $\mathrm{Pn}$, Gs, and Tr under both contrasting environments. PC2, however, 
mainly captured variance in RuBP or Ci under WW/DS environment (Fig. 2). Further analysis showed that pairwise Pearson correlations for six photosynthetic performances and three yield components yielded complementary information under both watering environments, and each corresponding trait posotively/negatively $(P<0.01$ or $P<0.05)$ correlating with four to eight other traits under single watering environment (Fig. 3), thus indicated that yield formation in maize was the result of the synergistic or inhibited effects of multiple photosynthetic performances under well-watered and droughtstressed environments, and the influence degress of drought stress to drought-sensitive maize plants photosynthesis and yield were larger. Moreover, $\mathrm{Pn}, \mathrm{Gs}, \mathrm{Ci}, \mathrm{RuBP}$ and WUE of the two $\mathrm{F}_{4}$ progeny populations depicted significantly positive correlation to female parent $(P<0.05$ or $P<0.01)$, respectively, $\mathrm{GW}$ and $\mathrm{KW}$ were significantly positive correlated to male parent $(P<0.05$ or $P<0.01)$, as well as $\mathrm{Tr}$ and $\mathrm{CW}$ showed significantly positive correlation to both parents $(P<0.05$ or $P<0.01)$ (Additional file 4: Table S2), indicative that the effects of both parents on different photosynthetic performances and yield components in $\mathrm{F}_{4}$ progeny populations were inconsistent.

\section{QTL analysis for photosynthetic performances under single watering environment}

To dissect the genetic control underlying six corresponding photosynthetic performances using single environment mapping with CIM, we totally mapped 54 QTLs ( 13 for Pn, 6 for Gs, 10 for Ci, 9 for Tr, 10 for RuBP, and 6 for WUE) across two $\mathrm{F}_{4}$ populations (POP-CT and POP-LT) based on both watering environments (WW and DS), and the phenotypic variance explained by individual QTL ranged from 2.37 (for RuBP in POP-LT under DS) to $18.21 \%$ (for $\mathrm{Ci}$ in POP-CT under DS) within each watering environment (Additional file 5: Fig. S3, Additional file 6: Table S3, Fig. 4). A total of 43 (79.63\%) of the identidied QTLs affecting six photosynthetic-related traits were detected under DS environments (Additional file 7: Fig. S4). For these identified QTLs, Pn, Gs, and RuBP displayed both additive (A) and non-additive (including PD, D, and OD) effects, however, QTLs for Ci, Tr, and WUE only showed no-nadditive effects (Additional file 5: Fig. S3). Moreover, approximately 33.33, 18.18, 37.50, 43.75, 35.29, and $44.44 \%$ alleles for increase in Pn, Gs, Ci, Tr, RuBP, and WUE were contributed by male parent TS141, respectively (Additional file 6: Table S3).

Joint analysis and QEIs for photosynthetic performances in multiple watering environments

The joint QTL analysis of all watering environments with MCIM revealed 54 QTLs controlling six photosynthetic-related traits in POP-CT and POP-CT, being absolutely equal to detected No. of QTLs based on single environment, and 25 of the identified QTLs were consistent with those of Pn, Gs, Ci, Tr, RuBP, and WUE through single environment mapping with CIM. These identified QTLs explained 2.07 (for $\mathrm{Ci}$ in POP-CT) to $13.21 \%$ (for Pn in POP-LT) phenotypic variance contributed by $h^{2}(A)$ (Fig. 4, Additional file 8: Table S4). Moreover, 24 QTLs (6 for Pn, 3 for Gs, 6 for Ci, 3 for Tr, 4 for RuBP, and 2 for WUE) were involved in QEI in two $\mathrm{F}_{4}$ populations, and accounted for 1.34 (for Ci in POP-LT) 6.86\% (for Pn inPOP-LT) of the phenotypic variance explained by $h^{2}(A E)$ (Additional file 8: Table S4). These QEI may thus impart stronger effects on photosynthetic performances in mazie under contrasting environments. In addition, for the two $\mathrm{F}_{4}$ populations, totally 7 stable bin intervals may be mediated by 
in Pn, Ci, and RuBP, bin 3.07_3.08 (umc1286/umc2275-umc2081) mapped a constitutive QEI for Gs, bin 6.05 (umc2040-bnlg1174a) found a constitutive QEI associated with $\mathrm{Ci}$ and RuBP, bin 7.00 (umc2177_umc1378) detected a constitutive QEI controlling Ci and RuBP, bin 7.02_7.04 (umc2057bnlg1666/umv1708) identified a constitutive QEI for Pn, bin 8.03 (bnlg1863-umc2075) dissected a constitutive QEI affecting WUE, bin 10.03 (bnlg1655-umc2016) validated a constitutive QEI involved in Pn and Tr (Additional file 8: Table S4).

cQTLs and candidate genes dissection for photosynthetic performances

Further analysis showed that 8 cQTLs were simultaneously identified by single environment mapping with CIM and joint analysis through MCIM in two $\mathrm{F}_{4}$ populations, which were located on chromosome 1 (Chr. 1), Chr. 6, Chr. 7, Chr. 8, and Chr. 10, and each cQTL accounting for $3.21 \sim 15.78 \%$ of the average observed phenotypic variance (Table 2). Except for the cQTL1 for WUE, other 7 cQTLs were detected under DS environments, indicative that these cQTLs regimes may have several stable alleles that were involved in photosynthesis under stress environments (Table 2). Moreover, 5 cQTLs, i.e., cQTL2 for Pn, Ci, Tr, and WUE, cQTL5 associated with Pn, Tr, $\mathrm{Ci}$, and RuBP, cQTL6 controlling Pn and RuBP, cQTL7 affecting Ci, Tr, and WUE, as well as cQTL8 involved in Pn, Tr, and RuBP (Table 2), imparted pleiotropic effect on two or four photosynthetic-related traits, suggesting that these cQTLs intervals control two or more tightly linked photosynthetic-related traits. In addition, the corresponding identified 8 cQTLs for all six photosynthetic-related traits were projected on the physical map B73 RefGen_v4 (http://www.maizegdb/gbrowse/mazie_V4), resulting in the identification of 17 candidate genes involved in leaf morphology and development, photosynthesis, and stress reponse (Table 2).

\section{Epistasis for photosynthetic performances}

Out of all QTLs identified, 6 and 8 pairs of epistatic interactions for six photosynthetic-related traits exhibited dominance-by-additive (DA) and dominance-by-dominance (DD) effects based on different watering environments in two $\mathrm{F}_{4}$ populations, and one for each pair epistatic interaction explained 2.11 (for Tr under WW) $4.96 \%$ (for Gs under WW) of the observed phenotypic variance contributed by $h^{2}(D A)$, and 2.09 (for Ci under DS) $5.87 \%$ (for Ci under DS) of the observed phenotypic variance contributed by $h^{2}(D D)$, respectively (Table 3, Fig. 5), indicating that the main effects of significant QTLs may be stronger on these six photosynthetic performances. Additionally, two pairs of the epistatic interactions were consistently detected in two $\mathrm{F}_{4}$ populations under different watering environments, i.e., simultaneously located between bin 1.07_1.10 (bnlg1025-mmc0041-phi308707) and bin 10.03 (bnlg1655-umc2016/umc1345) for Pn, and between bin 1.08_1.10 (mmc0041-phi308707) and bin 6.05 (umc2040-bnlg1174a) for Ci (Table 3, Fig. 5). The two epsistasis may thus be critical for MAS.

\section{Discussion}

\section{Maize photosynthetic performances variations in response to drought stress}

302 Approximately $95 \%$ of the organic matter accumulated by crops comes from its own photosynthesis, 
crop photsynthesis, and the responses of leaf photosynthesis to drought were mediated by two different physiological processes [35-36]. Firstly, SC and AMC decrease were recognized as the main driver of the photosynthetic response to drought stress, in order to reduce transpiration under water deprivations, the plant stomatal can close, and $\mathrm{SC}$ could limite $\mathrm{CO}_{2}$ diffusion efficiency from the atmosphere to the substomatal cavities to slow photosynthesis [37-38], and AMC rapidly decrease could further limite $\mathrm{CO}_{2}$ diffusion efficiency from the substomatal cavities to the chloroplast stroma during water stress [3940]. Secondly, photosynthesis could be limite by biochemical processes resulting in photosynthetic enzyme activity inhibition, Pn, Rubisco and RuBP activity decrease, etc. [7-9, 36]. Fortunately, above these findings were also supported by our results in this study, namely, drought-stressed Chang7-2, Langhuang, TS141, POP-CT, and POP-LT exhibited obvious decrease in Pn, Gs, Tr, and RuBP, their Ci and WUE, however, significantly displayed the increase relative of corresponding plants with sufficient water (Fig. 1, Additional file 2: Table S1). Furthermore, RuBP (average RC $18.96 \%$ ) and Gs (average $\mathrm{RC} 17.05 \%$ ) in response to drought stress were more sensitive than the response to Pn (average RC $16.02 \%$ ), Ci (average RC -9.34\%), Tr (average RC $10.80 \%$ ), and WUE (average RC -8.62 \%) (Fig. 1). It could be concluded that the drought-induced limitation of photosynthesis in maize was primarily due to $\mathrm{CO}_{2}$ diffusion efficiency from sub-stomatal interval internal cavities to carboxylation site in chloroplasts and degree of SC, and increasing evidences in maize also supported our results by Liu et al. [5],Veroneze-Júnior et al. [41], Perdomo et al. [42], and He et al. [4]. Therefore, genetic improvement of photosynthetic performances in maize can be applied to MAS breeding to improve drought tolerance and high-yielding in the future.

\section{Genetic architectures for photosynthetic performances}

Although a wealth of information from previous researches considerably improved our understanding of leaf photosynthetic performances [43-45], as well as applications in maize MAS breeding [5, 9, 18, 35], few studies considered the genetic basis of maize photosynthetic-related traits under water defict at the molecular level [20-22]. Based on the above considerations, in this study we detected 54 QTLs for six photosynthetic-related traits across two $\mathrm{F}_{4}$ populations via single watering environment mapping with CIM (Fig. 4, Additional file 6: Table S3), and for the identified QTLs, Pn, Gs, and RuBP showed both additive and non-additive effects under drought and non-drought stressed environments, but non-additive effects were largely responsible for the genetc basis of these three traits, of which accounted for 95.24, 81.82, and $94.12 \%$, respectively (Additional file 5: Fig. S3). However, all identified QTLs for Ci, Tr, and WUE displayed non-additive effects under both contrasting watering environments (Additional file 5: Fig. S3). These results were consistent with Wang and Zhang [18] and Li et al. [46] involved in photosynthetic-related traits in maize. Further Pearson correlation analysis among $\mathrm{F}_{4}$ progencies and their parents showed that $\mathrm{Pn}, \mathrm{Gs}, \mathrm{Ci}, \mathrm{RuBP}$, and WUE of the two $\mathrm{F}_{4}$ populations depicted significantly positive correlation to female parent, as well as $\operatorname{Tr}$ showed significantly positive correlation to both parents (Additional file 4: Table S2). Thereby, breeders should pay more attentation to the evaluation of $\mathrm{F}_{1}$ cross combinatons to make good use of their prominent non-additive effects and specific combining ability for above six photosynthetic-related traits, as well as should carefully select parents with elite 
stressed environments. Additionally, 43 of the 54 identified QTLs were found in drought-stressed environments (Additional file 8: Fig. S4). Suggesting that these identified QTLs controlling Pn, Gs, Ci, Tr, RuBP, and WUE could be changes under both contrasting environments, and the QTLs were identified under drought stress that may directly lead to differences in Pn, Gs, Ci, Tr, RuBP, and WUE.

GEI is critical in determining the adatation and fitness of genotypes in adverse environments, resulting in phenotypic variations [47-48], QEI information thus obtained was of great value for breeders and genetic researchers [47]. Furthermore, the variations of photosynthetic-related traits in two $\mathrm{F}_{4}$ populations showed that the wide variations, i.e. $\sigma_{g}^{2}, \sigma_{e}^{2}$, and $\sigma_{g e}^{2}$ were observed for six photosyntheticrelated traits $(P<0.01$ or $P<0.05)$, as well as their $h_{g e}^{2}$ ranged from $1.466 \%$ to $19.770 \%$ (Table 1 ). Further analysis of QEI in two $\mathrm{F}_{4}$ populations with MCIM among all watering environments also suggested that totally 24 QEIs (44.44 \%) of the identified joint QTLs controlling Pn, Gs, Ci, Tr, RuBP, and WUE were mapped, and each QEI explained $1.34 \sim 6.86 \%$ of the phenotypic variance by $h^{2}(A E)$ in present study (Additional file 8: Table S4). In this regard, photosynthetic-related traits are similar to other traits, such as yield-related, and leaf and inflorecence architecture traits in maize [27-29], of which showed extensive GEI. As a result, GEI may be a major challenge to MAS breeding for photosyntheticrelated traits in maize.

Epistasis, i.e. the interactions between genetic loci, is also thought to contribute to photosynthetic performances variations [49-51]. In maize, $\mathrm{Li}$ et al. [46] reported that SPAD was controlled by 2 major genes with $\mathrm{AD}$-epistatic effects and polygene with $\mathrm{AD}$ effects by mixed major gene plus polygene genetic model. Liu et al. [52] also suggested that SPAD was in agreement with the AD-epistatic model using diallel cross II (Griffing). As expected, in accord with previous studies [46, 52-53], totally 14 pairs of epistatic interactions with DA and DA controlling Pn, Gs, Ci, Tr, RuBP, and WUE based on different watering environments in two $\mathrm{F}_{4}$ populations, and which accounted for $2.09 \sim 5.87 \%$ of the observed phenotypic variance contributed by $h^{2}(D A / D D)$ that were clearly lower than those from A effects for all photosynthetic-related traits (Table 3, Fig. 5). It could be concluded that low contributions to phenotypic variance explained by DA/DD effects were due to large No. of DA/DD-QTLs with minor genetic effects, which would significantly influence the efficiency of MAS breeding for photosynrhetic-related traits. Notably, two stable DD-epistatic interactions were also validated in our study, i.e., controlling Pn between bin 1.07_1.08_1.10 (bnlg1025-mmc0041-phi308707) and bin 10.03 (bnlg1655umc2016/umc1345) was repeatedly detected in POP-CT under both contrasting watering environments/POP-LT under stressed environment, and affecting Ci between bin 1.08_1.10 (mmc0041phi308707) and bin 6.05 (umc2040-bnlg1174a) in two $F_{4}$ populations under both all four environments (Table 3, Fig. 5). These results were supported by Zhao et al. [26] and Sa et al. [54], who indicated that an $\mathrm{AD} /$ additive-additive (AA) epistatic interaction for $\mathrm{KW}$, plant height, and ear length, etc., located on Chr. 1 and Chr. 6/10 under multiple drought and non-drought environments. Hence, these regions of chromosomes may be considered as epistatic regulators that influence maize plant development, photosynthesis, and ear formation under multiple watering regimes. 
watering environments could provide guidance for fine mapping and maize MAS in the future. We totally identified 8 cQTLs for six photosynthetic-related traits using CIM/MCIM across POP-CT and POP-LT under multiple watering environments, of which accounting for $3.21 \sim 15.78 \%$ of the average observed phenotypic variance in this study (Table 2).

Af these, cQTL2 in bin 1.07_1.10 (bnlg1025/mmc0041-phi308707/umc1847) was simultaneously associated with $\mathrm{Pn}, \mathrm{Ci}$, Tr, and WUE, sQTL5 in bin 6.05 (umc2141-umc2040-bnlg1174a) was simultaneously involved in Pn, Tr, Ci, and RuBP, sQTL6 in bin 7.02_7.04 (umc2057-bnlg1666-umc1708) was simultaneously affected Pn and RuBP, sQTL7 in bin 8.03 (bnlg1863-umc2075) was simultaneously responsile for $\mathrm{Ci}$, Tr, and WUE, and sQTL8 in bin 10.03 (bnlg1655-umc2016/umc1345) was simultaneously associated with $\mathrm{Pn}, \mathrm{Tr}$, and RuBP in two $\mathrm{F}_{4}$ populations under both constracting watering environments, suggestive of pleiotropic cQTLs, which were highly agreed with the Pearson correlations analysis among six photosynthetic-related traits under both watering environments (Fig. 3). In bin 1.08_1.10 (umc83a-umc39c), in bin 6.05 (near gsy298e_pmg), bin $7.03 \_7.04$ (gsy113_cs-gsy107_pr), and bin 10.03 (gsy321_aba-gsy329_pp) intervals, Pelleschi et al. [21] also detected multiple QTLs associated with photosynthesis (i.e., sucrose-P synthase (SPS), hexoses (HEX), sucrose (SUC), AGP, Tr, and $\mathrm{CO}$ ) and leaf morphologies (i.e., number (LN), width (LW), length (LL), and relative water content (RWC)) across 120 F-2 $\times$ MBS847 RILs under drought and non-drought conditions. Li et al. [50] also mapped multiple QTLs involved in SPAD in bin 1.08 (umc1013-umc2047), 7.02 (umc1585-bnlg1305), bin 8.03 (bnlg1863-bnlg2046/umc2075-phi100175), and bin 10.03 (bnlg1655) regions in 172 Xu172 × Zong3 single segment substitution lines (SSSLs) under both high and low $\mathrm{N}$ treatments. Wang and Zhang [18] also identified two pleiotropic QTLs were simultaneously responsible for FCa, FCb, and FCt in bin 1.08 (mmc0041-bnlg1556) and bin 8.03 (umc1457-umc2199) using 189 A150-3-2 $\times$ Mo17 F2 plants under a single environment. Peng et al. [33] also found a QTL for grain tield per plant (GYPP) in near phi308707 (bin 1.10), a stable QTL controlled GYPP and kernel number per plant (KNPP) located in bnlg1094-bnlg1579 (bin 7.02_7.03), and a QTL affecting KW in bin 8.03 (bnlg1352-umc1778) cross 230 Qi319 $\times$ Huangzaosi and 235 Ye478 $\times$ Huangzaosi $F_{2: 3}$ families during six environments. These results indidated that pleiotropic cQTLs in the bin 1.07_1.10, 6.05, 7.02_7.04, 8.03, and 10.03 regions may play critical roles in leaf development, photosysthesis, and yield formation in maize under contrasting watering environments, and some important genes may be located in these bin regions. As expected, totally 11 cnadidate genes were also validated in above 5 pleiotropic cQTLs (cQTL 2, cQTL5, cQTL6, cQTL7, and cQTL8) intervals. GRMZM2G018627 (LHCB9) is a light harvesting chlorophyll binding (LHCB) protein, and Zhao et al. [55] proved that LHCB protein was required for the maintenance of photosystem I and specific protein-chlorophyll complexes especially under certain stress conditions. GRMZM2G162672 (chlg1), i.e., chlorophyll synthase G1 gene [56], acted as a chlorophyll biosynthetic process in biological progress via the AgBase v. 2.00 (http://agbase.arizona.edu/) online software with gene ontology (GO) analysis. GRMZM2G039113 (tan1), i.e., tangled 1 gene was required for spatial control of cytoskeletal arrays associated with cell division during maize leaf development [57], as well as the $\tan 1$ mutation could alter cell division orientations throught leaf development without altering leaf 
gene_center/gene). GRMZM5G809292 (PYG7), i.e., tetratricopeptide repeat domain-containing protein PYG7 [59], was the components of the chloroplast and thylakoid membrane in mellular component and involved in photosystem I assembly in biological progres by GO analysis. GRMZM2G042592, encoded the thioredoxin-like 6, chloroplast contained a variety of thioredoxin systems [60], while the thioredoxin could interact with CHLI subunits of $\mathrm{Mg}^{2+}$ chelatase to regulate the chelation of $\mathrm{Mg}^{2+}$ chelatase and chlorophyll synthesis precursor protoporphyrin IX [61]. Wang et al. [62] also predicted GRMZM2G042592 located in the same region of bnlg1863-umc2075 (bin 8.03), and its mutant with abnormal chloroplast, lacking pigment and reducing of PSII. GRMZM2G163437 (agps11), i.e., ADP glucose pyrophosphorylase small subunit leaf 1 gene, could be expressed in maize grain and source leaf during grain filling [63]. GRMZM2G033885 (psb29), encoded photosystem II subunit29, which was reversibly phosphorylated in maize upon exposure to high light in the cold condition, as well as its phosphorylation was controlled by the redox state of the plastoquinone pool, and may be part of a novel mechanism of photoprotection [64]. Another 2 candidate gens, i.e., GRMZM2G045431 (bHLH150) and GRMZM2G058451 (bHLH164), belonging to $b H L H$ transcription factors, which played important roles in maize grwoth and development, such as, root differentation [65], photomorphogenesis and light signal transduction [66], and stress response [67].

In addition, We also noted that cQTL3 affecting Gs in bin 3.07_3.08 (umc1286/umc2275-umc2081) with CIM/MCIM cross two $\mathrm{F}_{4}$ populations under drought and non-drought environments. Li et al. [50] also found a QEI for SPAD in bin 3.08 (umc1844-bnlg1182) under high N condition, and even Guo et al. [68] reported a meta-QTL (mQTL) for LW in bin 3.08 region from 28 original populations under multiple environments via mQTL analysis. Then further analysis validated two candidate genes, i.e., GRMZM2G159937 (bHIH57) and GRMZM2G117851 (bZIP1) in the bin 3.07_3.08 intervals in our study. GRMZM2G159937 may have all the functions of $b H L H$ transcription factor family [65-67]. Walsh et al. [69] reported liguleless2 (lg2) gene encoded a bZIP protein, involved in maize ligule and auricle development of leaf during vegetative and reproductive growth periods. GRMZM2G117851 was also a bZIP transcription factor, may play an important role in maize leaf development.

Additionally, 2 new cQTLs (cQTL1 and cQTL4) information were also found in our study. cQTL1 controlling WUE in bin 1.00_1.01 (bnlg149-bmc1014/umc1177) with CIM/MCIM cross both populations, of which GRMZM2G042250 (rld2, rolled leaf 2) was predicted, and its orthologous gene ATHB23 (i.e. a phytochrome B-interacting protein), was important for phytochrome B-mediated red light signaling in Arabidopsis thaliana [70]. cQTL4 responble for $\mathrm{Ci}$ in bin 4.08_4.09 (umc2041umc2188/umc2287) with CIM cross both populations under stressed and un-stressed environments. GRMZM2G446426 (MADS52), GRMZM2G038479 (bHLH8), and GRMZM2G074122 (pep3) were identified in cQTL4. GRMZM2G446426 as the MADS-transcription factor 52, could have the similar as ZmMADS4 in influencing chlorophyll content, and involve in response to osmotic stress [71]. GRMZM2G074122 was phosphoenolpyruvate carboxylase isoform 1 gene, which involved in carbon fixation and tricarboxylic acid cycle in biological process and influenced phosphoenolpyruvate carboxylase activity in molecular function via GO analysis. As a result, the 2 cQTLs intervals may provide new information for genetic basis dissection in maize photosynthetic performances under both watering environments in the future. 


\section{Conclusions}

Photosynthetic performances in maize were predominantly controlled by non-additive and QEIs effects, where more QEIs (87.5 \%) effects occurred in drought stress. 8 cQTLs (bin 1.00_1.01, bin 1.07_1.10, bin 3.07_3.08, bin 4.07_4.08, bin 6.05, bin 7.02_7.04, bin 8.03, and bin 10.03)affecting six photosynthetic-related traits could be useful for genetic improvement of these traits via QTL pyramiding, corresponding 5 cQTLs (cQTL2, cQTL5, cQTL6, cQTL7, and cQTL8) clusters indicated tight linkage or pleiotropy in the inheritance of these traits, and 17 candidate genes (GRMZM2G042250, GRMZM2G074122, GRMZM2G162672, GRMZM2G039113, GRMZM2G013657, GRMZM2G045431, and GRMZM2G163437) involved in leaf morphology and development, photosynthesis, and stress reponse coincided with above corresponding cQTLs.

\section{Supplementary information}

Additional file 1: Fig. S1. Statistics of the mean temperature (a), sun shine duration (b), rainfall (c), evaporation capacity (d), relative humidity (e), and average wind velocity ( $\mathrm{f}$ ) at the experimental site during the growing seasons (from April to September) in 2019, respectively, and statistics of the mean temperature (g), mean rainfall (h), mean evaporation capacity (i), and mean relative humidity (j) at the experimental site in recent 10 years (2009 2018), respectively

Additional file 2: Table S1 Statistics of photosynthetic-related traits in three parents, POP-LT, and POP-CT under different watering conditions, respectively

Additional file 3: Fig. S2 Frequency distribution of photosynthetic-related traits indifferent watering environments shown are Pn net photosynthetic rate (a, b), Gs stomatal conductance (c, d), Ci intercellular $\mathrm{CO}_{2}$ concentration (e, f), Tr transpiration rate (g, h), RuBP ribulose 1,5-biphospate carboxylase activity $\left(\mathrm{i}, \mathrm{j}\right.$ ), and WUE water use efficiency $(\mathrm{k}, \mathrm{l})$ of the two $\mathrm{F}_{2: 4}$ families (POP-CT and POP-LT) derived from the cross of Chang7-2 (CH) $\times$ TS141 (TS) and Langhuang (LH) $\times$ TS141 (TS) under well-watered (WW) and drought-stressed (DS) environments

Additional file 4: Table S2 Correlation coefficient of photosynthetic-related traits and yield components traits between progeny populations and parents

Additional file 5: Fig. S3 Summary of identified QTLs for photosynthetic-related traits (Pn net photosynthetic rate, Gs stomatal conductance, $\mathrm{Ci}$ intercellular $\mathrm{CO}_{2}$ concentration, $\mathrm{Tr}$ transpiration rate, RuBP ribulose 1,5-biphospate carboxylase activity, WUE water use efficiency) in two $\mathrm{F}_{4}$ populations (POP-CT and POP-LT) by single environment mapping with composite interval mapping (CIM). Including No. of QTLs and QTLs effects (A, additive effect; PD, partial dominance effect; D, dominance effect; OD, over-dominance effect), respectively

Additional file 6: Table S3 QTLs for photosynthetic-related traits were detected in POP-LT and POP-CT by single watering environment mapping with composite interval mapping (CIM)

Additional file 7: Fig. S4 Venn diagrams of identified QTLs comparison for Pn net photosynthetic rate (a and g), Gs stomatal conductance ( $b$ and h), Ci intercellular $\mathrm{CO}_{2}$ concentration ( $\mathrm{c}$ and $\mathrm{i}$ ), Tr transpiration rate ( $\mathrm{d}$ and j), RuBP ribulose 1,5-biphospate carboxylase activity (e and k), and WUE water use efficiency (f and 1 ) in two $\mathrm{F}_{4}$ populations (POP-CT and POP-LT) by single environment mapping with composite interval mapping (CIM). The identified QTLs in POP-CT under well-watered (green/blue circle) and drought-stressed (red/pink) environments, respectively

Additional file 8: Table S4 QTLs for photosynthetic-related traits were detected in POP-LT and POP-CT by joint analysis among all environments with a mixed linear model based on composite interval mapping (MCIM)

A, additive effect; AMC, apparent mesophyll conductance; $\mathrm{Ci}$, intercellular $\mathrm{CO}_{2}$ concentration; $\mathrm{CI}$, confidence interval; $\mathrm{CIM}$, composite interval mapping; cQTLs, constitutive QTLs, D, dominance effect; DA, dominance-by-additive effect; DD, dominanceby-dominance effect; Gs, stomatal conductance; GY, grain yield; MAS, marker-assisted selection; OD, over-dominance effect; PCA, principal component analysis; PD, partial dominance effect; Pn, net photosynthetic rate; QEIs, QTL $\times$ environment 
interactions; QTLs, quantitative trait loci; RuBP, ribulose 1,5-biphosphate carboxylase activity; SC, stomatal closure; Tr, transpiration rate; WUE, water use efficiency.

This work was supported by the Research Program Sponsored by Gansu Provincial Key Laboratory of Aridland Crop Science, Gansu Agricultural University, China (GSCS-2019-8), the Scientific Research Start-up Funds for Openly-recuited Doctors, Science and Technology Innovation Funds of Gansu Agricultural University, China (GAU-KYQD-2018-19; GAU-KYQD-2018-12), and the Developmental Funds of Innovation Capacity in Higher Education of Gansu, China (2019A-052; 2019A-054).

All relevant data are available within the manuscript and its additional files.

\section{Competing interests:}

The authors declare that they have no competing interests.

\section{Consent for publication}

Not applicable.

\section{Ethics approval and consent to participate}

Not applicable.

\section{Author details}

${ }^{1}$ Gansu Provincial Key Lab of Aridland Crop Science, Gansu Agricultural University, Lanzhou 730070, China. ${ }^{2}$ College of Agronomy, Gansu Agricultural University, Lanzhou 730070, China.

\section{References}

1. Messmer R, Fracheboud Y, Banziger M, Stamp P, Ribaut JM. Drought stress and tropical maize: QTL for leaf greenness, plant senescence, and root capacitance. Field Crop Res. 2011;124(1):93-103.

2. Chaves M, Davies B. Drought effects and water use efficiency: improving crop production in dry environments foreword. Funct Plant Bio. 2010;37(2):3-5.

3. Zhao XQ, Ren B, Peng YL, Xu MX, Fang P, Zhuang ZL, et al. Epistatic and QTL $\times$ environment interaction effects for ear related traits in two maize (Zea mays) populations under eight watering environments. Acta Agronomica Sinica. 2019;45(6):856-71.

4. He HJ, Kou SR, Wang XJ. Effects of drought stress on photosynthetic characteristics and yield components of different plant types of corn. Agri Res The Arid. 2011;29(3):63-6.

5. Liu J, Guo YY, Bai YW, Li HJ, Xue JQ, Zhang RH. Response of photosynthesis in maize to drought and re-watering. Russ J Plant Physiol. 2019;66(3):424-32.

6. Zhao XQ, Lu YT, Bai MX, Xu MX, Peng YL, Ding YF, et al. Response analysis of maize genotypes with different plant architecture to drought stress. Acta Prataculturae Sinica. 2019.

7. Zhang RH, Zhang XH, Camberato JJ, Xue JQ. Photosynthetic performance of maize hybrids to drought stress. Russ J Plant Physiol. 2015;62(6):788-96.

546 8. Tezara W, Marin O, Rengifo E, Martinez D, Herrara A. Photosynthesis and photoinhibition in two xerophytic shrubs during 
drought. Photosynthetica. 2005;43(1):37-45.

9. Liu J, Guo YY, Bai YW, Camberato JJ, Xue JQ, Zhang RH. Effects of drought stress on the photosynthesis in maize. Russ J Plant Physiol. 2018;65(6):849-56.

10. Pshenichnikova TA, Doroshkov AV, Osipova SV, Permyakov AV, Permyakova MD, Efimov VM, et al. Quantitative characteristics of pubescence in wheat (Triticum aestivum L.) are associated with photosynthetic parameters under conditions of normal and limited water supply. Planta. 2019;249(3):839-847.

11. Price AH, Young EM, Tomos AD. Quantitative trait loci associated with stomatal conductance, leaf rolling and heading date mapped in upland rice (Oryza sativa). New Phytol. 1997;137(1):83-91.

12. Sun J, Xie DW, Zhang EY, Zheng HL, Wang JG, Liu HL, et al. QTL mapping of photosynthetic-related traits in rice under salt and alkali stresses. Euphytica. 2019. 215(9):147.

13. Vivitha P, Raveendran M, Vijayalakshmi C, Vijayalakshmi D. Genetic dissection of high temperature stress tolerance using photosynthesis parameters in QTL introgressed lines of rice cv. Improved white Ponni. Ind J Plant Physiol. 2018;23(4):7417.

14. Herve D, Fabre F, Berrios EF, Leroux N, Chaarani GAI, Planchon C, et al. QTL analysis of photosynthesis and water status traits in sunflower (Helianthus annuus L.) under greenhouse conditions. J Exp Bot. 2001;52(362):1857-64.

15. Yan XY, Qu CM, Li JN, Chen L, Liu LZ. QTL analysis of leaf photosynthesis rate and related physiological traits in Brassica napus. J Integr Agr. 2015;14(7):1261-8.

16. Levi A, Lianne O, Paterson AH, Saranga Y. Photosynthesis of cotton near-isogenic lines introgressed with QTLs for productivity and drought related traits. Plant Sci. 2009;177(2):88-96.

17. Zheng JY, Oluoch G, Riaz Khan MK, Wang XX, Cai XY, Zhou ZL, et al. Mapping QTLs for drought tolerance in an $F_{2: 3}$ population from an inter-specific cross between Gossypium tomentosum and Gossypium hirsutum. Genetics Mol Res. 2016;15(3):1-14.

18. Wang AY, Zhang CQ. QTL mapping for chiprophyll content in maize. Hereditas. 2008;30(8):1083-91.

19. Trachsel S, Messmer R, Stamp P, Ruta N, Hund A. QTLs for early vigor of tropical maize. Mol Breed. 2010;25(1):91-103.

20. Yu TT, Liu CX, Mei XP, Wang JG, Wang GQ, Cai YL. Correlation and QTL analyses for photosynthetic traits in maize. J Southwest Uni. 2015;37(9):1-10.

21. Pelleschi S, Leonardi A, Rocher JP, Cornic G, de-Vienne D, Thevenot C,et al. Analysis of the relationships between growth, photosynthesis and carbohydrate metabolism using quantitative trait loci (QTLs) in young maize plants subjected to water deprivation. Mol Breed. 2006;17(1):21-39.

22. Prado SA, Cabrera-Bosquet L, Grau A, Coupel-Ledru A, Millet EJ, Welcker C, et al. Phenomics allows identification of genomic regions affecting maize stomatal conductance with conditional effects of water deficit and evaporative demand. Plant Cell Environ. 2018;41(2):314-26.

23. Qi XL, Li Y, Wang YM, Han LP, Zhang L, Hu L, et al. Development of transgenic wheat lines with ZmPPDK gene and analysis on its photosynthetic and yield characteristics. J Triticeae Crops. 2019;39(8):950-7.

24. Ishimaru K, Ohkawa Y, Ishige T, Tobias DJ, Ohsugi R. Elevated pyruvate, orthophosphate dikinase (PPDK) activity alters carbon metabolism in $\mathrm{C}_{3}$ transgenic potato with a $\mathrm{C}_{4}$ maize $P P D K$ gene. Physiologia Plantarum. 1998;103(3):340-6.

25. Ishimaru K, Ichikawa $\mathrm{H}$, Matsuoka M, Ohsugi R. Analysis of a $\mathrm{C}_{4}$ maize pyruvate, orthophosphate dikinase expressed in $\mathrm{C}_{3}$ transgenic Arabidopsis plants. Plant Sci. 1997;129(1):57-64.

26. Zhao XQ, Zhang JW, Fang P, Peng YL. Comparative QTL analysis for yield components and morphological traits in maize (Zea mays L.) under water-stressed and well-watered conditions. Breed Sci. 2019; doi:10.1270/jsbbs.18021

27. Zhao XQ, Fang P, Zhang JW, Peng YL. QTL mapping for six ear leaf architecture traits under water-stressed and well-watered conditions in maize (Zea mays L.). Plant Breed. 2018;137(1):60-72.

28. Zhao XQ, Peng YL, Zhang JW, Fang P, Wu BY. Identification of QTLs and meta-QTLs for seven agronomic traits in multiple maize populations under well-watered and water-stressed conditions. Crop Sci. 2018;58(2):507-20.

29. Zhao XQ, Peng YL, Zhang JW, Fang P, Wu BY. Mapping QTLs and meta-QTLs for two inflorescence architecture traits in multiple maize populations under different watering environments. Mol Breed. 2017;37(7):91.

30. Khan MA, Tong F, Wang WB, He JB, Zhao TJ, Gai JY. Analysis of QTL-allele system conferring drought tolerance at seedling stage in a nested association mapping population of soybean [Glycine max (L.) Merr.] using a novel GWAS procedure. Planta. 2018;248(4):947-62.

31. Wang S, Basten CJ, Zeng ZB. Windows QTL Cartographer 2.5. Department of Stattistics. Available: http://statgen.ncsu.edu/ 
qtlcart/winqtlcart.htm. 2010 .

32. Stuber SW, Edwards MD, Wendel J. $\mathrm{F}_{1}$ molecular marker facilitated investigations of quantitative trait loci in maize. II. Factors influencing yield and its component traits. Crop Sci. 1987;27(4):639-48.

33. Peng B, Wang Y, Li YX, Liu C, Zhang Y, Liu ZZ, et al. Correlation analysis and conditional QTL analysis of grain yield and yield components in maize. Acta Agronomica Sinica. 2010;36(10):1624-33.

34. Semagn K, Beyene Y, Warburton ML, Tarekegne A, Mugo S, Meisel B, et al. Meta-analysis of QTL for grain yield and anthesis silking interval in 18 maize populations evaluated under water-stress and well-watered environments. BMC Genomics. 2013;14:313.

35. Zhang YW, Zhao XG, Guan ZB, Hou JL, Wang XF, Dong YH, et al. High photosynthetic-efficiency germplasm screening of crops: Research Progress. Chinese Agri Sci Bull. 2019;35(18):1-11.

36. Zhou L, Wang SQ, Chi YG, Li QK, Huang K, Yu QZ. Response of photosynthetic parameters to drought in subtropical forsest ecosystem of China. Sci Rep. 2015;5:18254.

37. Egea G, Verhoef A, Vidale PL. Towards an improved and more flexible representation of water stress in coupled photosynthesis stomatal conductance models. Agr. Forest Meteorol. 2011;151:1370-84.

38. Grassi G, Magnani F. Stomatal, mesophyll conductance and biochemical limitations to photosynthesis as affected by drought and leaf ontogeny in ash and oak trees. Plant Cell Environ. 2005;28(7):834-49.

39. Flexas J, Barbour MM, Brendel O, Cabrera HM, Carriqui M, Diaz-Espejo A, et al. Mesophyll diffusion conductance to $\mathrm{CO}_{2}$ : An unappreciated central player in photosynthesis. Plant Sci. 2012;193-194:70-84.

40. Flexas J, Ribas-Carbo M, Diaz-Espejo A, Galmes J, Medrano H. Mesophyll conductance to $\mathrm{CO}_{2}$ : current knowledge and future prospects. Plant Cell Environ. 2008;31(5):602-21.

41. Veroneze-Júnior V, Martins M, Mc-Leod L, Souza KRD, Santos-Filho PR, Magalhaes PC, et al. Leaf application of chitosan and physiological evaluation of maize hybrids contrasting for drought tolerance under water restriction. Braz J Biol. 2019; doi:10.1590/1519-6984.218391

42. Perdomo JA, Capo-Bauca S, Carmo-Silva E, Galmes J. Rubisco and Rubisco activise play an important role in the biochemical limitations of photosynthesis in rice, wheat, and maize under high temperature and water deficit. Front Plant Sci. 2017;8:490.

43. Shi DK, Yao TL, Liu NN, Deng M, Duan HY, Wang LL, et al. Genome-wide association study of chlorophyll content in maize. Scientia Agri Sinica. 2019;52(11):1839-57.

44. Yonemaru JI, Miki K, Choi S, Kiyosawa A, Goto K. A genomic region harboring the Pl1 alllele from the peruvian cultivar JC072A confers purple cob on Japanese flint corn (Zea mays L.). Breed Sci. 2018;68(5):582-86.

45. Zaida PH, Rashid Z, Vinayan MT, Almeida GD, Phagna RK, Babu R. QTL mapping of agronomic waterlogging tolerance using recombinant inbred lines derived from tropical maize (Zea mays L.). PLoS ONE. 2015;10(4):e0124350.

46. Li HT, Xu HY, Li JF, Zhu Q, Chi M, Wang J. Analysis of gene effect on chlorophyll content in maize. Crops. 2019;5:46-51.

47. Li SS, Wang JK, Zhang LY. Inclusive composite interval mapping of QTL by environment interactions in Biparental populations. PLoS ONE. 2015;10(7):e0132414.

48. Ungerer MC, Halldorsdottir SS, Purugganan MD, Mackay TFC. Genotype environment interactions at quantitative trait loci affecting inflorescence development in Arabidopsis thaliana. Genetics. 2003;165(1):353-65.

49. Bhoite R, Onyemaobi I, Si P, Siddique KHM, Yan GJ. Identification and validation of QTL and their associated genes for pre-emergent metribuzin tolerance in hexaploid wheat (Triticum aestivum L.). BMC Genetics. 2018;19(1):102.

50. Li YD, Wang Y, Tang H, Xu H, Tan JF, Han YL. QTL mapping for ear leaf stay-green in maize under high and low N conditions. J Plant Nutr Fertilizers. 2019;25(1):115-22.

51. Li YL, Wang XY, Huang SY, Cheng L, Ni ZQ, Wang YN, et al. QTL mapping and epistatic interaction analysis of canopy parameters in soybean. Mol Plant Breed. 2016;14(12):3414-30.

52. Liu PF, Jiang F, Chen QC, Zeng MH, Zhang Y, Zhang ZL, et al. Genetic analysis on chlorophyll content of leaf in fresheatable sweet corn. J Anhui Agri Uni. 2013;40(1):134-8.

53. Zhao YM, Yan M, Xu HW, Zhou DS, Teng SS, Liu DY, et al. Analysis of genetic main effects and genotype-environment interactions of leaf stay-green in maize. Chinese Agri Sci Bull. 2008;24(1):164-7.

54. Sa KJ, Park JY, Woo SY, Ramekar RV, Jang CS, Lee JK. Mapping of QTL traits in corn using a RIL population derived from a cross of dent corn $\times$ waxy corn. Genes Genom. 2015;37(1):1-24.

55. Zhao L, Cheng DM, Huang XH, Chen M, Osto LD, Xing JL, et al. A light harvesting complex-like protein in maintenance 
of photosynthetic components in Chlamydomonas. Plant Physiol. 2017;174(4):2419-33.

56. Walley JW, Sartor RC, Shen ZX, Schmitz RJ, Wu KJ, Urich MA, Integration of omic networks in a developmental atlas of maize. Sci. 2016;353(6301):814-8.

57. Cleary AL, Smith LG. The tangled1 gene is required for spatial control of cytoskeletal arrays associated with cell division during maize leaf development. Plant Cell. 1998;10(11):1875-88.

58. Smith LG, Hake S, Sylvester AW. The tangled-1 mutation alters cell division orientations throught maize leaf development without altering leaf shape. Development. 1996;122(2):481-9.

59. Hoopes GM, Hamilton JP, Wood JC, Esteban E, Pasha A, Vaillancourt B, et al. An updated gene atlas for maize reveals organspecific and stress-induced genes. Plant J.2019;97(6):1154-67.

60. Schurmann P, Buchanan BB. The ferredoxin/thioredoxin system of oxygenic photosynthesis. Antioxid Redox Sign. 2008;10(7):1235-74.

61. Luo T, Fan T, Liu Y, Rothbart M, Yu J, Zhou S, et al. Thioredoxin redox regulates ATPase activity of magnesium chelatase CHLI subunit and moddulates redox-mediated signaling in tetrapyrrole biosynthesis and homeostasis of reactive oxygen species inpea plants. Plant Physiol. 2012;159(1):118-30.

62. Wang F, Duan SM, Li T, Wang RN, Tao YS. Fine mappping and candidate gene analysis of leaf color mutant in maize. J Plant Genetic Resource. 2018;19(6):1205-9.

63. Prioul JL, Jeannette E, Reyss A, Gregory N, Giroux M, Hannah LC, et al. Expression od ADP-glucose pyrophosphorylase in maize (Zea mays L.) grain and source leaf during grain filling. Plant Physiol. 1994;104(1):179-87.

64. Bergantino E, Sandona D, Cugini D, Bassi R. The photosystem II subunit CP29 can be phosphorylated in both $\mathrm{C}_{3}$ and $\mathrm{C}_{4}$ plants as suggested by sequence analysis. Plant Mol Biol. 1998;36(1):11-22.

65. Ramsay NA, Glover BJ. Myb-Bhlh-WD40 protein complex and the evolution of cellular diversity. Trens Plant Sci. 2005;10(2):63-70.

66. Mo XT, Zhao J, Fan YL, Wang L. Research progress on structure and function of maize transcription factors. J Agri Sci Technol. 2013;15(3):7-17.

67. Li Z, Gao Q, Liu Y, Overexpression of transcription factor ZmPTF1 improves low phosphate tolerance of maize by regulating carbon metabolism and root growth. Planta. 2011;233(6):1129-43.

68. Guo SL, Zhang J, Qi JS, Yue RQ, Han XH, Yan SF, et al. Analysis of meta-quantitative trait loci and their candidate genes related to leaf shape in maize. Chinese Bull Bot. 2018;53(4):487-501.

69. Walsh J, Freeling M. The liguleless 2 gene of maize functions during the transition from the vegetative to the reproductive shoot apex. Plant J. 1999;19(4):489-95.

70. Choi H, Jeong S, Kim DS, Na HJ, Ryu JS, Lee SS, et al. The homeodomain-leucine zipper ATHB23, a phytochrome Binteracting protein, is important for phytochrome B-mediated red light signaling. Physiol Plant. 2014;150(2):308-20.

71. Schreiber DN, Bantin J, Dresselhaus T. The MADS box transcription factor ZmMADS2 is required for anther and pollen maturation in maize and accumulates in apoptotic bodies during anther dehiscence. Plant Physoil. 2004;134(3):1069-79. 
Fig. 1 The rate of changes of photosynthetic-related traits (Pn net photosynthetic rate, Gs stomatal conductance, Ci intercellular $\mathrm{CO}_{2}$ concentration, $\mathrm{Tr}$ transpiration rate, RuBP ribulose 1,5-biphospate carboxylase activity, WUE water use efficiency) and yield component traits (EW ear weight, GW grain weight per ear, KW 100-kernel weight) in three parents (LH Langhuang, CH Chang72, TS TS141) and two $\mathrm{F}_{4}$ families (POP-LT, POP-CT) under different watering conditions (WW well-watered environment at Wuwei in 2019, DS, drought-stressed environment at Wuwei in 2019), $F$-value**/* indicated the significant difference at $P<0.01$ or $P<0.05$ level via one-way analysis of variance (ANOVA)

Fig. 2 Principal component analysis (PCA) of photosynthetic-related traits (Pn net photosynthetic rate, Gs stomatal conductance, $\mathrm{Ci}$ intercellular $\mathrm{CO}_{2}$ concentration, Tr transpiration rate, RuBP ribulose 1,5-biphospate carboxylase activity, WUE water use efficiency) in the both $\mathrm{F}_{4}$ populations (POP-LT, POP-CT) under different watering environments, (a, b) Eigenvalues of principal components (PCs) under contrasting watering environments, respectively, PCs with eigenvalue greater than 1 were retained, (c, d) The effects of corresponding photosynthetic-related traits in PC1 and PC2 under contrasting watering environments, (e, f) Eigenvectors of photosynthetic-related traits in PC1 and PC2 under contrasting watering environments

Fig. 3 Pearson correlation among corresponding tested traits (Pn net photosynthetic rate, Gs stomatal conductance, Ci intercellular $\mathrm{CO}_{2}$ concentration, Tr transpiration rate, RuBP ribulose 1,5-biphospate carboxylase activity, WUE water use efficiency; EW ear weight, GW grain weight per ear, KW 100-kernel weight) under well-watered (a) and drought-stressed environments. Red/sapphire dotted lines designated positive /negative correlations between both traits $(P<0.01)$, and black/gray dotted lines designated positive/negative correlations between both traits $(P<0.05)$, respectively. Circles of different sizes reflected No. of corresponding tested traits

Fig. 4 Genetic map and identified QTLs for photosynthetic-related traits (Pn net photosynthetic rate, Gs stomatal conductance, Ci intercellular $\mathrm{CO}_{2}$ concentration, Tr transpiration rate, RuBP ribulose 1,5-biphospate carboxylase activity, WUE water use efficiency) in two $\mathrm{F}_{4}$ populations (POP-CT and POP-LT) by single environment mapping with compositive interval mapping (CIM) and joint analysis of all environments with mixed-linear-model-based composite interval mapping (MCIM). Green/red and sapphire/pink rectangular, circle, triangle, rhombus, hexagon, and pentagram represented identified QTLs for Pn, Gs, Ci, Tr, RuBP, and WUE under well-watered/drought-stressed environment in POP-CT and POP-LT with CIM, respectively. Yellow and blue rectangular, circle, triangle, rhombus, hexagon, and pentagram represented identified QTLs for Pn, Gs, Ci, Tr, RuBP, and WUE in POP-CT and POP-LT among all watering environments with MCIM, respectively.

Fig. 5 Epistasis of QTLs were identified for photosynthetic-related traits in POP-CT (a) and POP-LT (b) by joint analysis among all environments with mixed-linear-model-based composite interval mapping (MCIM). Black/brown dotted lines represented dominance-by-additive/dominance (DA/DD) epistatic interaction effects, respectively. Green/red and sapphire/pink rectangular, circle, triangle, rhombus, hexagon, and pentagram represented corresponding QTLs for net photosynthetic rate (Pn), stomatal conductance (Gs), intercellular $\mathrm{CO}_{2}$ concentration $(\mathrm{Ci})$, transpiration rate ( $\mathrm{Tr}$ ), ribulose 1,5-biphospate carboxylase activity (RuBP), and water use efficiency (WUE) under well-watered/drought-stressed environment in POP-CT and POP-LT, respectively 
717 Table 1 Variance analysis, broad-sense heritability $\left(h^{2}\right)$, and genotype $\times$ environment interaction (GEI) heritability $\left(h_{g e}^{2}\right)$ of the photosynthetic-related traits in the POP-LT and POP-CT, respectively

\begin{tabular}{|c|c|c|c|c|c|c|}
\hline Item & Pn & Gs & $\mathrm{Ci}$ & $\operatorname{Tr}$ & RuBP & WUE \\
\hline Corrected model & $279.722 * *$ & $0.025^{*}$ & $9400.878 * *$ & $4.289 * *$ & $0.033 * *$ & $3.883 * *$ \\
\hline Intercept & $5687.46^{* *}$ & $0.262 * *$ & $212617.821^{* *}$ & $232.181 * *$ & $0.713^{* *}$ & $329.913 * *$ \\
\hline$\sigma_{g}^{2}$ & $102.911^{* *}$ & $0.013^{*}$ & $3214.928 * *$ & $5.129 * *$ & $0.032 * *$ & $3.728 * *$ \\
\hline$\sigma_{e}^{2}$ & $71.213^{* *}$ & $0.007^{*}$ & $837.813 * *$ & $2.259 * *$ & $0.016^{* * *}$ & $1.039^{*}$ \\
\hline$\sigma_{g e}^{2}$ & $25.598 * *$ & $0.008^{*}$ & $320.051 * *$ & $2.293 * *$ & $0.009 *$ & $0.126^{*}$ \\
\hline$\sigma_{\varepsilon}^{2}$ & $2.514 * *$ & $0.002 \mathrm{NS}$ & $31.353 \mathrm{NS}$ & $0.217 \mathrm{NS}$ & $0.002 \mathrm{NS}$ & $0.101 \mathrm{NS}$ \\
\hline Total & 6573.387 & 0.293 & 222050.052 & 236.688 & 0.749 & 333.897 \\
\hline$h^{2}$ & 88.939 & 48.148 & 91.030 & 69.683 & 68.817 & 86.778 \\
\hline$h_{g e}^{2}$ & 9.977 & 14.815 & 4.531 & 15.576 & 9.677 & 1.466 \\
\hline Corrected model & $395.355^{* *}$ & $0.124^{*}$ & $6224.776^{* *}$ & $8.165 * *$ & $0.024 * *$ & $3.480 * *$ \\
\hline Intercept & $6174.345^{* *}$ & $0.268^{* *}$ & $182666.913^{* *}$ & $288.320 * *$ & $1.138 * *$ & $407.828 * *$ \\
\hline$\sigma_{g}^{2}$ & $121.67 * *$ & $0.016^{*}$ & $3014.544 * *$ & $6.087 * *$ & $0.020 * *$ & $3.401 * *$ \\
\hline$\sigma_{e}^{2}$ & $53.986^{* *}$ & $0.009^{*}$ & $731.405^{* *}$ & $6.573 * *$ & $0.012 * *$ & $1.020^{*}$ \\
\hline$\sigma_{g e}^{2}$ & $29.726 *$ & $0.010^{*}$ & $363.211^{* *}$ & $3.266^{* *}$ & $0.004 *$ & $1.059 *$ \\
\hline$\sigma_{\varepsilon}^{2}$ & $3.687 \mathrm{NS}$ & $0.009 \mathrm{NS}$ & $24.168 \mathrm{NS}$ & $0.108 \mathrm{NS}$ & $0.002 \mathrm{NS}$ & $0.199 \mathrm{NS}$ \\
\hline Total & 6573.387 & 0.301 & 188915.857 & 296.593 & 1.163 & 411.507 \\
\hline$h^{2}$ & 78.513 & 46.429 & 90.882 & 73.692 & 62.500 & 69.049 \\
\hline$h_{g e}^{2}$ & 9.591 & 8.929 & 5.475 & 19.770 & 6.250 & 10.750 \\
\hline
\end{tabular}

719 Pn net photosynthetic rate, Gs stomatal conductance, Ci intercellular $\mathrm{CO}_{2}$ concentration, Tr transpiration rate, RuBP ribulose 1,5-

720 biphospate carboxylase activity, WUE water use efficiency, $\sigma_{g}^{2}$ the genotypic variance, $\sigma_{e}^{2}$ the environmental variance, $\sigma_{g e}^{2}$ the

721 'genotypic $\times$ environment' interaction variance, $\sigma_{\varepsilon}^{2}$ the error variance, $h^{2}$ heritability, $h_{g e}^{2}$ the genotype by environment

722 interaction heritability. **/* indicated the significant difference at the $P<0.01 / P<0.05$ probability level, respectively 
Table 2 Summary of constitutive QTLs (cQTLs) and candidate genes for photosynthetic-related traits in the POP-LT and POP-CT, respectively

\begin{tabular}{|c|c|c|c|c|c|c|c|}
\hline cQTL & Marker interval & Trait & Population (Environment) & QTL & Bin & $R^{2}$ (Average) (\%) & Candidate gene (Annotation) \\
\hline cQTL1 & $\begin{array}{c}\text { bnlg149- } \\
\text { bmc1014/umc1177 }\end{array}$ & WUE & POP-CT (WW), POP-LT (Joint) & qWUE-Ch.1-1, qWUE-J1-1 & $1.00-1.01$ & $2.53-3.88(3.21)$ & GRMZM2G042250 (rld2) \\
\hline \multirow{4}{*}{ cQTL2 } & & Pn & POP-CT (WW, DS, Joint), POP-LT (DS) & qPn-Ch.1-2 & \multirow{4}{*}{ 1.07/1.08_1.10 } & $5.68-9.33(7.06)$ & \multirow{4}{*}{ GRMZM2G018627 (LHCB9) } \\
\hline & bnlg1025/mmc0041- & $\mathrm{Ci}$ & POP-CT (WW, DS, Joint), POP-LT (DS, Joint) & qCi-Ch.1-1 & & $5.98-10.13(7.16)$ & \\
\hline & phi308707/umc1847 & $\operatorname{Tr}$ & POP-CT (DS, WW), POP-LT (DS) & qTr-Ch.1-1 & & 3.77-9.35 (5.88) & \\
\hline & & WUE & POP-CT (DS, Joint), POP-LT (WW, DS) & qWUE-Ch.1-2, qWUE-J1-2 & & 2.92-7.99 (4.95) & \\
\hline cQTL3 & $\begin{array}{l}\text { umc1286/umc2275- } \\
\text { umc2081 }\end{array}$ & Gs & POP-CT (WW, DS, Joint), POP-LT (WW, DS, Joint) & qGs-Ch.3-1 & 3.07-3.08 & $4.14-10.10(7.93)$ & $\begin{array}{c}\text { GRMZM2G159937 (bHLH57), GRMZM2G117851 } \\
(\text { bZIPI) }\end{array}$ \\
\hline cQTL4 & $\begin{array}{c}\text { umc2041- } \\
\text { umc2188/umc2287 }\end{array}$ & $\mathrm{Ci}$ & POP-CT (WW, DS), POP-LT (WW, DS) & qCi-Ch.4-1 & 4.08_4.09 & $5.98-13.40(10.68)$ & $\begin{array}{c}\text { GRMZM2G446426 (MADS52), GRMZM2G038479 } \\
\text { (bHLH8), GRMZM2G074122 (pep3) }\end{array}$ \\
\hline \multirow{4}{*}{ cQTL5 } & & Pn & POP-CT (WW, DS) & qPn-Ch.6-1 & \multirow{4}{*}{6.05} & $6.76-9.53(8.15)$ & \\
\hline & umc2141-umc2040- & $\operatorname{Tr}$ & POP-CT (WW, DS, Joint) & qTr-Ch.6-1 & & $3.58-8.69(5.43)$ & GRMZM2G162672 (chlgI), GRMZM2G039113 (tanI), \\
\hline & bnlg1174a & $\mathrm{Ci}$ & POP-CT (Joint), POP-LT (Joint) & qCi-J6-1 & & 3.83-3.95 (3.89) & GRMZM2G013657 (dwill) \\
\hline & & RuBP & POP-LT (WW, DS, Joint) & qRuBP-Ch.6-1 & & 2.64-3.38 (3.00) & \\
\hline cQTL6 & $\begin{array}{l}\text { umc2057- } \\
\text { bnlg1666/umc1708 }\end{array}$ & $\begin{array}{c}\text { Pn } \\
\text { RuBP }\end{array}$ & $\begin{array}{l}\text { POP-CT (WW, DS, Joint), POP-LT (WW, DS, Joint) } \\
\text { POP-CT (WW, DS, Joint), POP-LT (Joint) }\end{array}$ & $\begin{array}{c}\text { qPn-Ch.7-2 } \\
\text { qRuBP-Ch.7-1, qRuBP-J7-1 }\end{array}$ & 7.02_7.04 & $\begin{array}{l}5.03-17.26(9.95) \\
3.73-7.84(5.56)\end{array}$ & $\begin{array}{c}\text { GRMZM2G045431 (bHLH150), GRMZM2G033885 } \\
\text { (psb29), GRMZM2G058451 (bHLH164), } \\
\text { GRMZM5G809292 (PYG7) }\end{array}$ \\
\hline \multirow{3}{*}{ cQTL7 } & \multirow{3}{*}{ bnlg1863-umc2075 } & $\mathrm{Ci}$ & POP-CT (WW, DS), POP-LT (WW, DS, Joint) & qCi-Ch.8-1 & \multirow{3}{*}{8.03} & $11.74-18.21(15.78)$ & \\
\hline & & $\operatorname{Tr}$ & POP-CT (WW, DS), POP-LT (WW, DS, Joint) & qTr-Ch.8-1 & & $3.48-8.69(5.42)$ & GRMZM2G042592 (Similar to Thioredoxin-like 6) \\
\hline & & WUE & POP-CT (Joint), POP-LT (Joint) & qWUE-J8-1 & & $4.05-7.53(5.79)$ & \\
\hline \multirow{3}{*}{ cQTL8 } & \multirow{3}{*}{$\begin{array}{c}\text { bnlg1655- } \\
\text { umc2016/umc1345 }\end{array}$} & Pn & POP-CT (Joint), POP-LT (WW, Joint) & qPn-J10-1, qPn-Ch.10-1 & \multirow{3}{*}{10.03} & $6.10-11.17(8.78)$ & \multirow{3}{*}{$\begin{array}{c}\text { GRMZM2G042895 (bHLH116), GRMZM2G163437 } \\
\text { (agpsll) }\end{array}$} \\
\hline & & $\operatorname{Tr}$ & POP-CT (WW, DS, Joint), POP-LT (WW, DS, Joint) & qTr-Ch.10-1 & & $2.92-5.25(3.65)$ & \\
\hline & & RuBP & POP-LT (WW, DS) & qRuBP-Ch.10-1 & & 3.16-4.02(3.59) & \\
\hline
\end{tabular}

724 Pn net photosynthetic rate, Gs stomatal conductance, $\mathrm{Ci}$ intercellular $\mathrm{CO}_{2}$ concentration, Tr transpiration rate, RuBP ribulose 1,5-biphospate carboxylase activity, WUE water use efficiency 
Table 3 Epistatic interactions for photosynthetic-related traits were detected in POP-LT and POP-CT with a mixed linear model based on composite interval mapping (MCIM)

\begin{tabular}{|c|c|c|c|c|c|c|c|c|c|c|c|}
\hline Trait & Environment & QTL(i) & Marker interval(i) & $\operatorname{Bin}(i)$ & QTL(j) & Marker interval(j) & $\operatorname{Bin}(j)$ & DA & DD & $\mathrm{h}^{2}(\mathrm{DA})(\%)$ & $\mathrm{h}^{2}(\mathrm{DD})(\%)$ \\
\hline \multirow[t]{2}{*}{ Pn } & WW & qPn-Ch.1-2 & mmc0041-phi308707 & $1.08 \_1.10$ & qPn-J10-1 & bnlg1655-umc2016 & 10.03 & & -0.61 & & 4.62 \\
\hline & DS & qPn-Ch.1-2 & mmc0041-phi308707 & 1.08_1.10 & qPn-J10-1 & bnlg1655-umc2016 & 10.03 & & -0.74 & & 5.35 \\
\hline Gs & WW & qGs-Ch.3-1 & umc1286-umc2081 & 3.07 & qGs-J4-1 & umc2041-umc2188 & 4.08 & 0.05 & & 4.96 & \\
\hline \multirow[t]{4}{*}{$\mathrm{Ci}$} & WW & qCi-Ch.1-1 & mmc0041-phi308707 & $1.08 \_1.10$ & qCi-J6-1 & umc2040-bnlg1174a & 6.05 & & -1.18 & & 3.56 \\
\hline & DS & qCi-Ch.1-1 & mmc0041-phi308707 & $1.08 \_1.10$ & qCi-J6-1 & umc2040-bnlg1174a & 6.05 & & -1.40 & & 4.01 \\
\hline & WW & qCi-J6-1 & umc2040-bnlg1174a & 6.05 & qCi-J7-1 & umc2177-umc1378 & 7.00 & -0.98 & & 2.79 & \\
\hline & DS & qCi-J6-1 & umc2040-bnlg1174a & 6.05 & qCi-J7-1 & umc2177-umc1378 & 7.00 & -1.07 & & 3.01 & \\
\hline \multirow[t]{2}{*}{$\operatorname{Tr}$} & WW & qTr-Ch.5-1 & umc2216-umc1072 & $5.06 \_5.07$ & $\mathrm{qTr}-\mathrm{Ch} .10-1$ & bnlg1655-umc2016 & 10.03 & 0.06 & & 2.11 & \\
\hline & DS & qTr-Ch.5-1 & umc2216-umc1072 & $5.06 \_5.07$ & qTr-Ch.10-1 & bnlg1655-umc2016 & 10.03 & 0.08 & & 2.64 & \\
\hline \multirow[t]{2}{*}{ RuBP } & WW & qRuBP-J1-1 & mmc0041-phi308707 & $1.08 \_1.10$ & qRuBP-J7-1 & umc2177-umc1378 & 7.00 & & -0.05 & & 3.73 \\
\hline & DS & qRuBP-J1-1 & mmc0041-phi308707 & $1.08 \_1.10$ & qRuBP-J7-1 & umc2177-umc1378 & 7.00 & & -0.09 & & 4.80 \\
\hline \multirow[t]{2}{*}{ WUE } & WW & qWUE-Ch.1-2 & mmc0041-phi308707 & $1.08 \_1.10$ & qWUE-J8-1 & bnlg1863-umc2075 & 8.03 & -0.38 & & 3.13 & \\
\hline & DS & qWUE-Ch.1-2 & mmc0041-phi308707 & 1.08_1.10 & qWUE-J8-1 & bnlg1863-umc2075 & 8.03 & -0.32 & & 2.94 & \\
\hline Pn & DS & qPn-J1-1 & bnlg1025-mmc0041 & 1.07_1.08 & qPn-Ch.10-1 & bnlg1655-umc1345 & 10.03 & & -0.43 & & 2.46 \\
\hline \multirow[t]{3}{*}{$\mathrm{Ci}$} & DS & qCi-Ch.1-1 & mmc0041-phi308707 & 1.08_1.10 & qCi-J6-1 & umc2040-bnlg1174a & 6.05 & & -1.96 & & 5.87 \\
\hline & WW & qCi-J4-1 & umc1963-umc1031 & 4.04_4.05 & qCi-J6-1 & umc2040-bnlg1174a & 6.05 & & 1.25 & & 3.39 \\
\hline & DS & qCi-J4-1 & umc1963-umc1031 & 4.04_4.05 & qCi-J6-1 & umc2040-bnlg1174a & 6.05 & & -1.03 & & 2.09 \\
\hline \multirow[t]{4}{*}{$\operatorname{Tr}$} & wW & qTr-J6-1 & bnlg238-umc2310 & 6.00 & qTr-J9-1 & dupssr29-umc2359 & 9.07 & & -0.10 & & 5.04 \\
\hline & DS & qTr-J6-1 & bnlg238-umc2310 & 6.00 & qTr-J9-1 & dupssr29-umc2359 & 9.07 & & -0.11 & & 5.18 \\
\hline & WW & $\mathrm{qTr}-\mathrm{Ch} .10-1$ & bnlg1655-umc1345 & 10.03 & qTr-J10-1 & bnlg1839-bnlg2162 & 10.07_10.08 & & 0.09 & & 4.75 \\
\hline & DS & qTr-Ch.10-1 & bnlg1655-umc1345 & 10.03 & qTr-J10-1 & bnlg1839-bnlg2162 & $10.07 \_10.08$ & & 0.06 & & 3.10 \\
\hline RuBP & WW & qRuBP-J1-1 & mmc0041-phi308707 & $1.08 \_1.10$ & qRuBP-Ch.6-1 & umc2040-bnlg1174a & 6.05 & 0.05 & & 2.47 & \\
\hline
\end{tabular}


at Wuwei in 2019, DS drought-stressed environment at Wuwei in 2019, DA the dominance by additive epistatic interaction effects, DD the dominance by dominance epistatic interaction effects, $h^{2}(D A)$ percentage of phenotypic variance explained by the dominance-by-additive epistatic interaction effects, $h^{2}(D D)$ percentage of phenotypic variance explained by the dominance-by-dominance epistatic interaction effects 
Figures

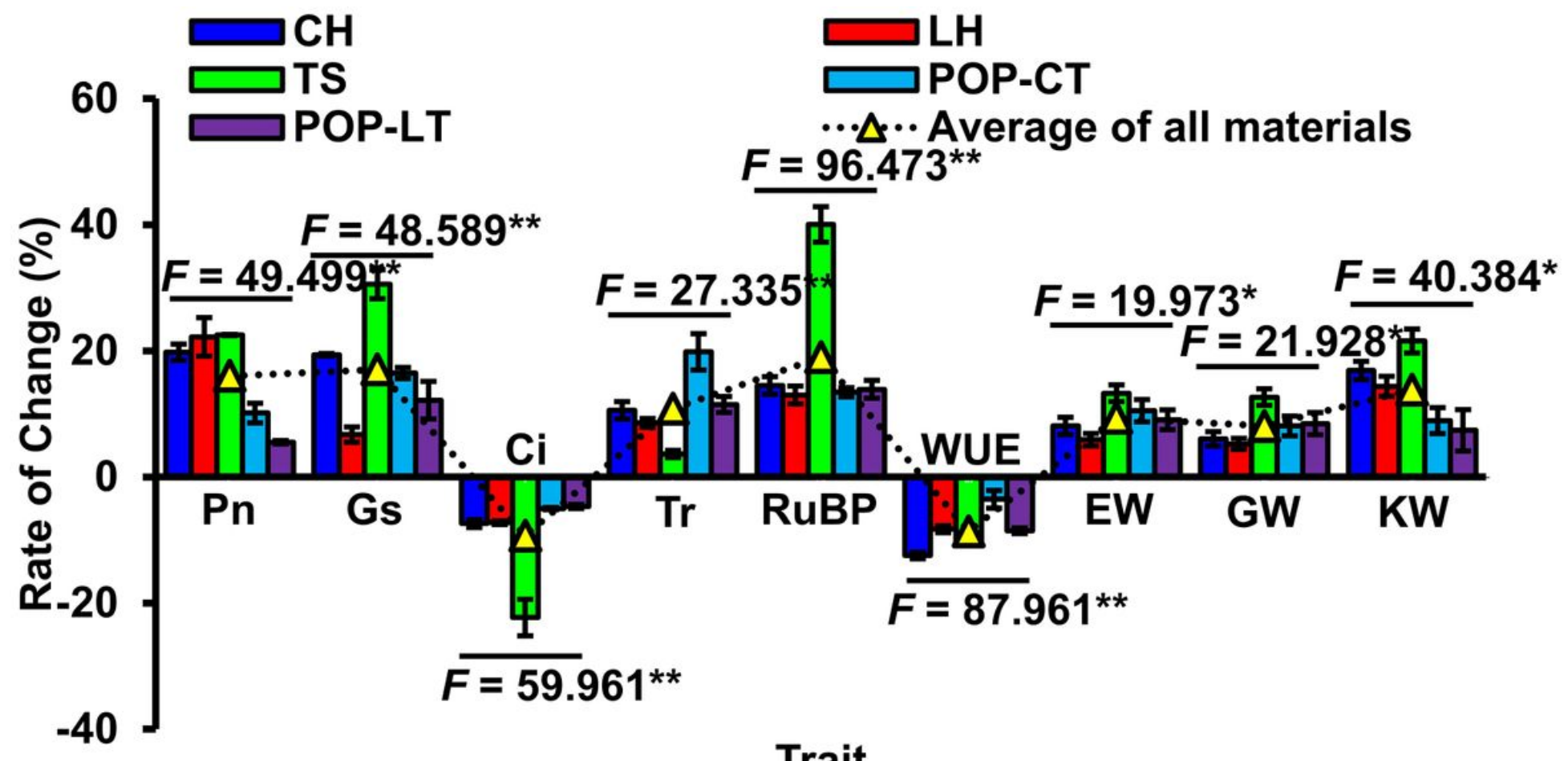

Figure 1

The rate of changes of photosynthetic-related traits (Pn net photosynthetic rate, Gs stomatal conductance, $\mathrm{Ci}$ intercellular $\mathrm{CO} 2$ concentration, $\mathrm{Tr}$ transpiration rate, RuBP ribulose 1,5-biphospate carboxylase activity, WUE water use efficiency) and yield component traits (EW ear weight, GW grain weight per ear, KW 100-kernel weight) in three parents (LH Langhuang, CH Chang7-2, TS TS141) and two F4 families (POP-LT, POP-CT) under different watering conditions (WW well-watered environment at Wuwei in 2019, DS, drought-stressed environment at Wuwei in 2019), F-value ${ }^{\star \star} / *$ indicated the significant difference at $\mathrm{P}<0.01$ or $\mathrm{P}<0.05$ level via one-way analysis of variance (ANOVA) 
Scree Plot
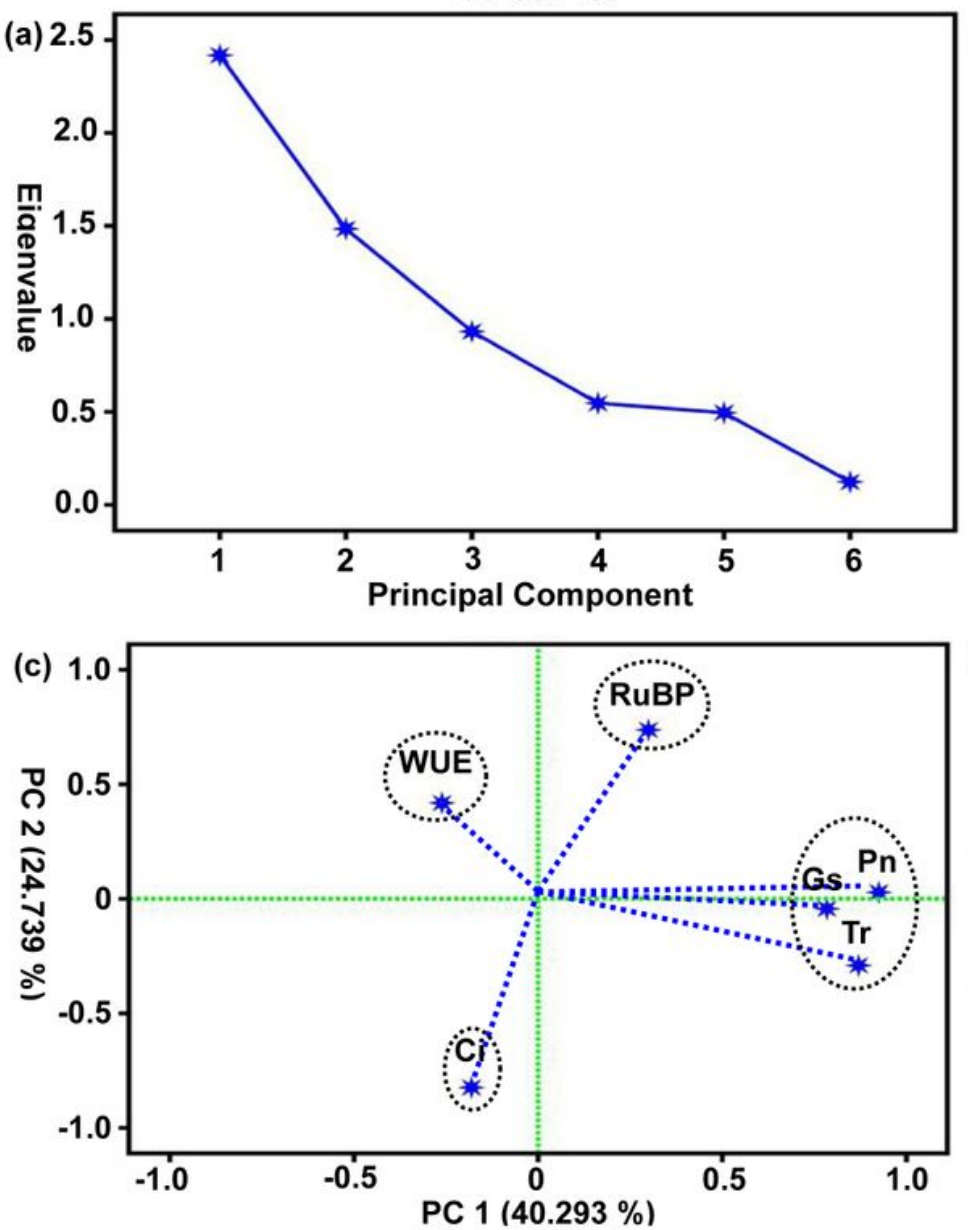

(e) -Component $1-$ Component 2

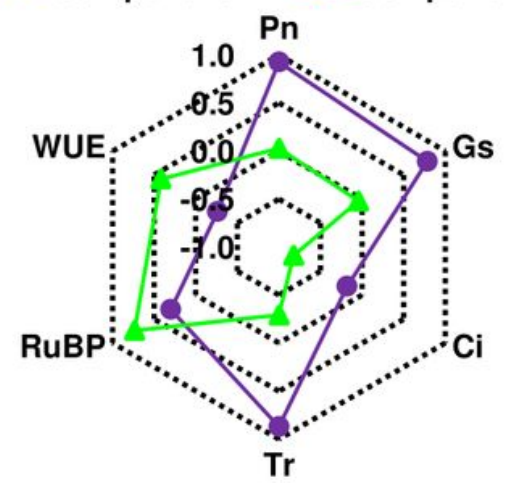

Scree Plot
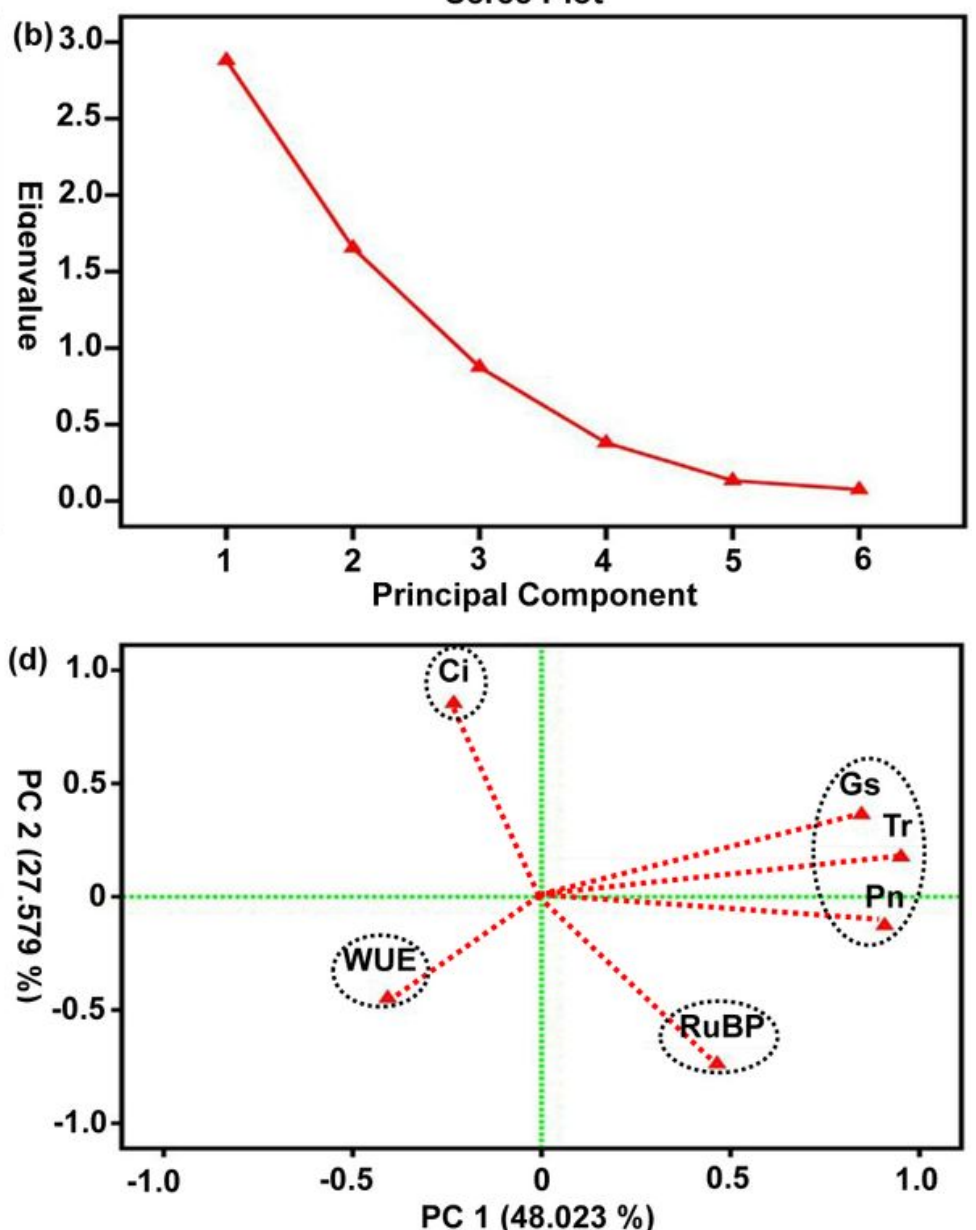

(f)

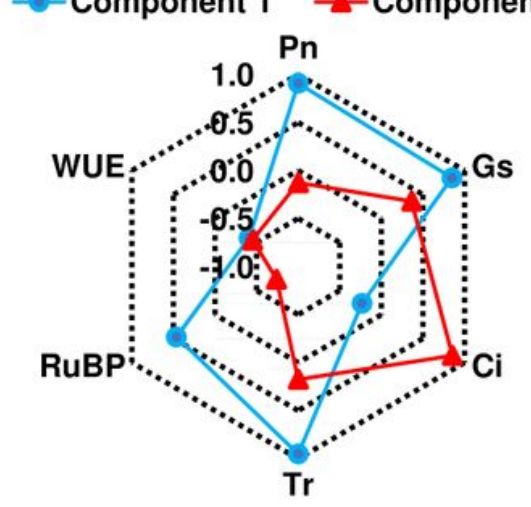

Figure 2

Principal component analysis (PCA) of photosynthetic-related traits (Pn net photosynthetic rate, Gs stomatal conductance, $\mathrm{Ci}$ intercellular $\mathrm{CO} 2$ concentration, $\mathrm{Tr}$ transpiration rate, RuBP ribulose 1,5biphospate carboxylase activity, WUE water use efficiency) in the both F4 populations (POP-LT, POP-CT) under different watering environments, $(a, b)$ Eigenvalues of principal components (PCs) under contrasting watering environments, respectively, PCs with eigenvalue greater than 1 were retained, $(c, d)$ The effects of corresponding photosynthetic-related traits in PC1 and PC2 under contrasting watering environments, $(e, f)$ Eigenvectors of photosynthetic-related traits in PC1 and PC2 under contrasting watering environments 

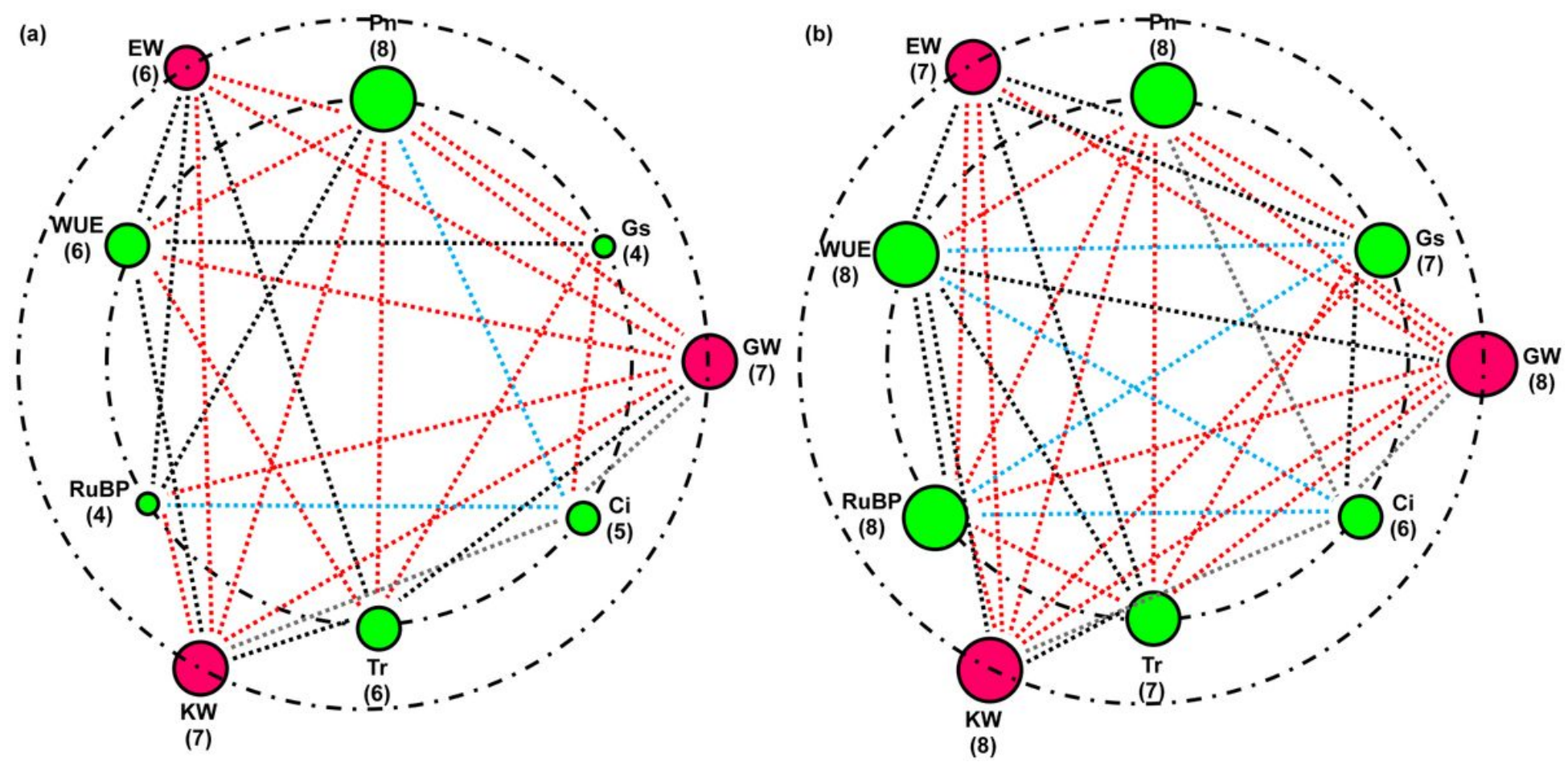

Figure 3

Pearson correlation among corresponding tested traits (Pn net photosynthetic rate, Gs stomatal conductance, $\mathrm{Ci}$ intercellular $\mathrm{CO} 2$ concentration, $\mathrm{Tr}$ transpiration rate, RuBP ribulose 1,5-biphospate carboxylase activity, WUE water use efficiency; EW ear weight, GW grain weight per ear, KW 100-kernel weight) under well-watered (a) and drought-stressed environments. Red/sapphire dotted lines designated positive /negative correlations between both traits $(P<0.01)$, and black/gray dotted lines designated positive/negative correlations between both traits $(P<0.05)$, respectively. Circles of different sizes reflected No. of corresponding tested traits 

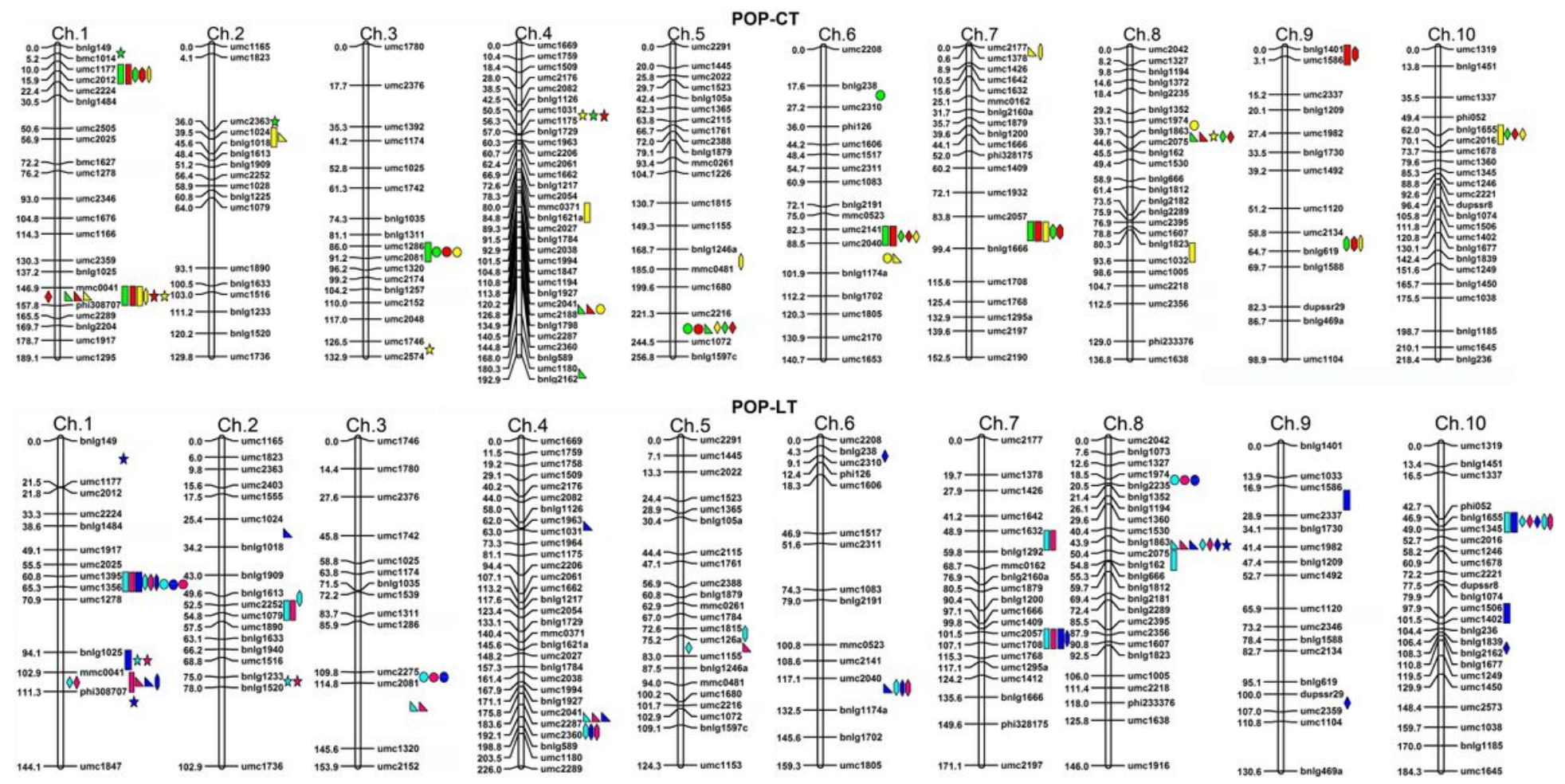

Figure 4

Genetic map and identified QTLs for photosynthetic-related traits (Pn net photosynthetic rate, Gs stomatal conductance, $\mathrm{Ci}$ intercellular $\mathrm{CO} 2$ concentration, $\mathrm{Tr}$ transpiration rate, RuBP ribulose 1,5biphospate carboxylase activity, WUE water use efficiency) in two F4 populations (POP-CT and POP-LT) by single environment mapping with compositive interval mapping (CIM) and joint analysis of all environments with mixed-linear-model-based composite interval mapping (MCIM). Green/red and sapphire/pink rectangular, circle, triangle, rhombus, hexagon, and pentagram represented identified QTLs for Pn, Gs, Ci, Tr, RuBP, and WUE under well-watered/drought-stressed environment in POP-CT and POP-LT with $\mathrm{CIM}$, respectively. Yellow and blue rectangular, circle, triangle, rhombus, hexagon, and pentagram represented identified QTLs for Pn, Gs, Ci, Tr, RuBP, and WUE in POP-CT and POP-LT among all watering environments with $\mathrm{MCIM}$, respectively.

(a)

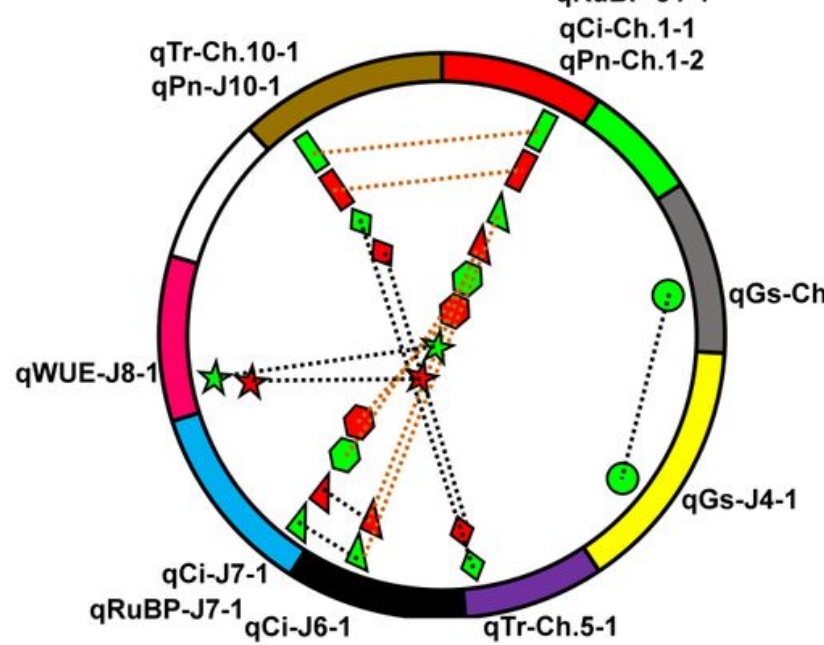

(b)

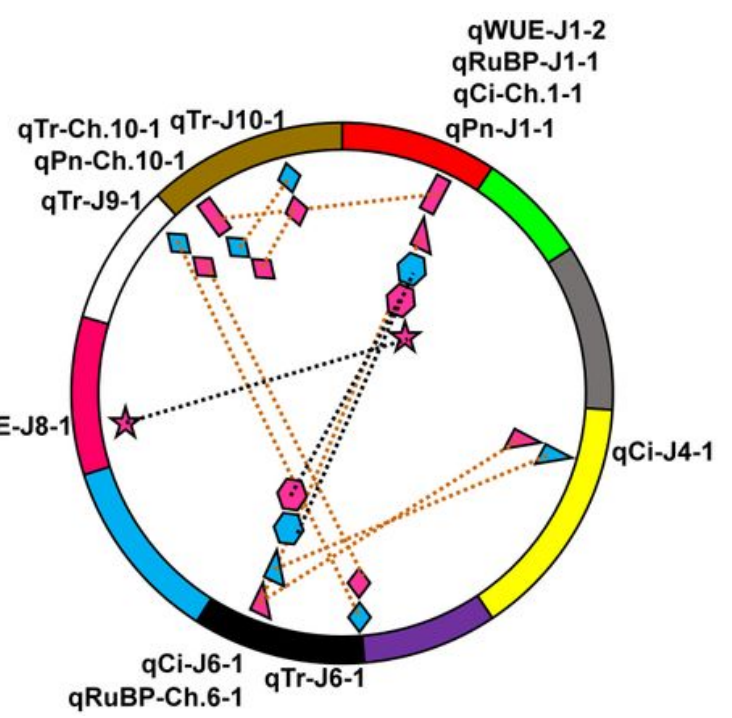

口Ch.1

口Ch.2

Ch.3

口Ch.4

口Ch.5

घCh.6

$\square$ Ch.7

aCh.8

口Ch.9

口Ch.10 


\section{Figure 5}

Epistasis of QTLs were identified for photosynthetic-related traits in POP-CT (a) and POP-LT (b) by joint analysis among all environments with mixed-linear-model-based composite interval mapping (MCIM). Black/brown dotted lines represented dominance-by-additive/dominance (DA/DD) epistatic interaction effects, respectively. Green/red and sapphire/pink rectangular, circle, triangle, rhombus, hexagon, and pentagram represented corresponding QTLs for net photosynthetic rate (Pn), stomatal conductance (Gs), intercellular $\mathrm{CO} 2$ concentration (Ci), transpiration rate $(\mathrm{Tr})$, ribulose 1,5-biphospate carboxylase activity (RuBP), and water use efficiency (WUE) under well-watered/drought-stressed environment in POP-CT and POP-LT, respectively

\section{Supplementary Files}

This is a list of supplementary files associated with this preprint. Click to download.

- Additionalfile3.docx

- Additionalfile1.docx

- Additionalfile6.docx

- Additionalfile8.docx

- Additionalfile7.docx

- Additionalfile4.docx

- Additionalfile5.docx

- Additionalfile2.docx 